\title{
Auxiliary Hamiltonian representation of the nonequilibrium Dyson equation
}

\author{
Karsten Balzer and Martin Eckstein \\ Max Planck Research Department for Structural Dynamics, University of Hamburg-CFEL, 22607 Hamburg, Germany
}

(Received 4 December 2013; published 31 January 2014)

\begin{abstract}
The nonequilibrium Dyson (or Kadanoff-Baym) equation, which is an equation of motion with a long-range memory kernel for real-time Green functions, underlies many numerical approaches based on the Keldysh formalism. In this paper we map the problem of solving the Dyson equation in real time onto a noninteracting auxiliary Hamiltonian with additional bath degrees of freedom. The solution of the auxiliary model does not require the evaluation of a memory kernel and can thus be implemented in a very memory efficient way. The mapping is derived for a self-energy which is local in space and is thus directly applicable within nonequilibrium dynamical mean-field theory (DMFT). We apply the method to study the interaction quench in the Hubbard model for an optical lattice with a narrow confinement, using inhomogeneous DMFT in combination with second-order weak-coupling perturbation theory. We find that, although the quench excites pronounced density oscillations, signatures of the two-stage relaxation similar to the homogeneous system can be observed by looking at the time-dependent occupations of natural orbitals.
\end{abstract}

DOI: 10.1103/PhysRevB.89.035148

PACS number(s): 71.27.+a, 71.10.Fd, 05.30.-d, 05.70.Ln

\section{INTRODUCTION}

The field of strongly correlated materials out of equilibrium is a rapidly growing research area. On the one hand, this is due to ultrafast pump-probe experiments which allow one to coherently control and manipulate solids in a time-resolved fashion by external laser fields. Examples in this direction include quantum interference effects in photoexcited Mott insulators [1], light-induced superconductivity in cuprates [2], and experiments on ultrafast magnetism [3]. On the other hand, ultracold atomic gases confined in optical lattices [4] allow one to study fundamental condensed-matter models for strongly correlated quantum systems in great detail, e.g., [5], and independently of any lattice imperfections. In theory, the investigation of correlated systems out of equilibrium has revealed novel relaxation phenomena such as doublon decay [6], prethermalization [7-10], and dynamical transitions [10-13].

The microscopic description of correlated systems out of equilibrium requires appropriate quantum statistical methods. A promising approach is provided by nonequilibrium dynamical mean-field theory (DMFT) [14,15], which works well for higher-dimensional systems and becomes exact in the limit of infinite dimensions. Other approaches include, e.g., cluster perturbation theory [17], linked cluster expansions [16], the nonequilibrium dual fermion approach [18], and nonequilibrium self-energy functional theory [19]. All these methods are based on the Keldysh formalism [20] and involve a Dyson equation which describes the time evolution of a quantum many-body system in terms of the one-particle nonequilibrium Green function and a corresponding self-energy [21]. In general, the self-energy introduces time retardation effects, which render the numerical solution of the Dyson equation in nonequilibrium a complicated task in itself. Therefore, when translational invariance is lost, the solution is restricted to either short times or to a small number of orbitals (or bands). Only with a massively parallelized time evolution comprising distributed memory [22] or with further approximations such as the generalized Kadanoff-Baym ansatz [23] have these limitations been overcome so far.
The idea of the present paper is to develop an alternative method to solve the Dyson equation, which can be efficient and computationally less demanding when the self-energy is sufficiently local in space. The approach builds on recent work [24] where it was shown that the action of nonequilibrium DMFT can be mapped onto a single-impurity Anderson model by fitting the hybridization function of the DMFT bath. The present paper discusses how a similar decomposition of the self-energy defines a noninteracting auxiliary Hamiltonian which, on the one hand, couples to additional bath orbitals but, on the other hand, leads to the same one-particle nonequilibrium Green function as the interacting many-body problem we start from. The key to an efficient time propagation algorithm lies in the fact that the auxiliary system involves no interactions, such that the corresponding Green function is subject to simple Markovian dynamics and can be determined by exact diagonalization techniques. Furthermore, the decomposition of the self-energy is causal (i.e., the time-dependent parameters of the auxiliary problem depend only on the self-energy at earlier times), such that the mapping can easily be incorporated into approaches like nonequilibrium DMFT, where the self-energy is given as a functional of the Green function itself.

The paper is organized as follows. In Secs. II A and II B, we describe the theoretical framework, elucidate the Dyson equation for the study of nonequilibrium situations, define the auxiliary Hamiltonian, and formulate the conditions for a valid mapping. Section II C illustrates the mapping within the Hubbard-I approximation, and Sec. II D explains in detail the decomposition of the self-energy and the determination of the parameters in the auxiliary system. Section IIE then gives details on the computation of the Green function of the auxiliary model (see also Appendixes A and B). Thereafter, in Sec. III, we test the matrix decomposition of the selfenergy for small Hubbard clusters (Sec. III A), illustrate the time propagation of the auxiliary system (Sec. III A) and investigate the scalability of the method to long times. Finally, Sec. IV contains our main application. Here, we study the relaxation dynamics of the Fermi-Hubbard model following 
an interaction quench, with a particular focus on the effects of an optical trap. A summary is presented in Sec. V.

\section{THEORY}

\section{A. Nonequilibrium Dyson equation}

Our main objective is to describe the time evolution of an interacting quantum many-body system which is initially (at time $t=0$ ) in thermodynamic equilibrium at temperature $T=$ $\beta^{-1}$ and evolves unitarily under a time-dependent Hamiltonian $H(t)$ for times $t>0$. As prototype we consider the single-band Hubbard model

$$
\begin{aligned}
H(t)= & \sum_{i j \sigma} J_{i j}(t) c_{i \sigma}^{\dagger} c_{j \sigma}+\sum_{i \sigma}\left[V_{i}(t)-\mu\right] n_{i \sigma} \\
& +U(t) \sum_{i}\left(n_{i \uparrow}-\frac{1}{2}\right)\left(n_{i \downarrow}-\frac{1}{2}\right),
\end{aligned}
$$

where $c_{i \sigma}^{\dagger}\left(c_{i \sigma}\right)$ are creation (annihilation) operators for an electron with spin $\sigma$ on site $i$ of the lattice, $J_{i j}$ denotes the hopping amplitude between sites $i$ and $j, V_{i}$ is an external potential, $\mu$ the chemical potential, $n_{i \sigma}=c_{i \sigma}^{\dagger} c_{i \sigma}$ is the density, and $U$ is the local Coulomb interaction.

Using nonequilibrium Green-function techniques, the time evolution of the Hubbard model (1) is determined by the Dyson equation

$$
G\left(t, t^{\prime}\right)=G_{0}\left(t, t^{\prime}\right)+\int_{\mathcal{C}} d s \int_{\mathcal{C}} d \bar{s} G_{0}(t, s) \Sigma(s, \bar{s}) G\left(\bar{s}, t^{\prime}\right),
$$

where the matrix elements of $G$ are the one-particle nonequilibrium Green functions of system (1) defined on the L-shaped Keldysh time contour $\mathcal{C}$,

$$
\begin{aligned}
G_{i j \sigma}\left(t, t^{\prime}\right) & =-i\left\langle T_{\mathcal{C}} c_{i \sigma}(t) c_{j \sigma}^{\dagger}\left(t^{\prime}\right)\right\rangle \\
& =-i \frac{\operatorname{tr}\left[T_{\mathcal{C}}\left\{\exp (S) c_{i \sigma}(t) c_{j \sigma}^{\dagger}\left(t^{\prime}\right)\right\}\right]}{\operatorname{tr}\left[T_{\mathcal{C}}\{\exp (S)\}\right]},
\end{aligned}
$$

with action $S=-i \int_{\mathcal{C}} d t H(t)$ and contour-ordering operator $T_{\mathcal{C}}$ (see, e.g., $[15,21,25,26]$ for an introduction into the Keldysh technique; our notation for contour functions, integrals, and differentials follows [15]). Similarly, $G_{0}$ denotes the noninteracting Green functions $G_{0, i j \sigma}\left(t, t^{\prime}\right)=-i\left\langle T_{\mathcal{C}} c_{i \sigma}(t) c_{j \sigma}^{\dagger}\left(t^{\prime}\right)\right\rangle_{0}$, evaluated from Eq. (3) with $U=0$, and $\Sigma$ denotes the self-energy with elements $\Sigma_{i j \sigma}\left(t, t^{\prime}\right)$.

The self-energy is typically determined by the Green function in a self-consistent way. Within DMFT, for example, $\Sigma\left(t, t^{\prime}\right)$ is obtained from the solution of a single-impurity Anderson model with a bath that is determined by the lattice Green function. In perturbation theory, the self-energy $\Sigma$ is given by a series of Feynman diagrams and appears as a functional of $G$ and the interaction $U$. Important conservation laws such as density, energy, and momentum conservation are in particular obeyed for any truncation of the derivative $\Sigma_{i j \sigma}\left(t, t^{\prime}\right)=\delta \Phi / \delta G_{j i \sigma}\left(t^{\prime}, t\right)$, where $\Phi[G, U]$ denotes the Luttinger-Ward functional [27]. Simple examples are the Hartree-Fock or second Born approximation which are of first and second order in the interaction, respectively.

For a given self-energy, the numerical solution of Eq. (2) can be performed in different ways. One possibility is to discretize all quantities on the time contour $\mathcal{C}$ and to apply standard matrix inversion techniques to determine $G$ [28]. More frequently Eq. (2) is transformed into a set of integrodifferential equations (the Kadanoff-Baym equations [21]) which are then solved within a time propagation scheme; see [22,29-33]. The transformation of Eq. (2) to differential form is achieved by using the equation of motion for $G_{0}$,

$$
\sum_{r}\left[\delta_{i r}\left(i \partial_{t}+\mu\right)-h_{i r}(t)\right] G_{0, r j \sigma}\left(t, t^{\prime}\right)=\delta_{i j} \delta_{\mathcal{C}}\left(t, t^{\prime}\right),
$$

where $h_{i j}$ defines the single-particle part of the Hamiltonian, i.e., the quadratic part of Eq. (1) is given by $H_{0}(t)=$ $\sum_{i j \sigma}\left(h_{i j}(t)-\mu\right) c_{i \sigma}^{\dagger} c_{j \sigma}$. In combination with (2), Eq. (4) gives

$$
\begin{aligned}
& \sum_{r}\left\{\left[\delta_{i r}\left(i \partial_{t}+\mu\right)-h_{i r}(t)\right] G_{r j \sigma}\left(t, t^{\prime}\right)\right. \\
& \left.-\int_{\mathcal{C}} d s \Sigma_{i r \sigma}(t, s) G_{r j \sigma}\left(s, t^{\prime}\right)\right\}=\delta_{i j} \delta_{\mathcal{C}}\left(t, t^{\prime}\right) .
\end{aligned}
$$

This equation clearly reveals the non-Markovian structure inherent to the Dyson equation: The differential $\partial_{t} G\left(t, t^{\prime}\right)$ depends on the value of $G$ at different times, and $\Sigma$ takes the role of a memory kernel. For a self-consistent determination of $\Sigma$ and $G$, the time propagation of $G$ with Eq. (5) and the determination of $\Sigma$ from $G$ can be iterated until convergence successively on each time step. A severe restriction for the numerical solution of this equation is the memory needed to store the functions $G_{i j \sigma}\left(t, t^{\prime}\right)$ for all times on the contour.

\section{B. Auxiliary Hamiltonian}

The central idea of the present paper is to avoid a memory kernel in the time propagation scheme for the nonequilibrium Green functions. To this end, we will map the interacting system (1) onto a larger auxiliary system [denoted $H_{\text {aux }}(t)$ ] which is noninteracting and the Green function of which consequently obeys simple Markovian dynamics. The auxiliary system must be constructed such that its single-particle Green functions exactly equal the solutions of the Dyson equation (2) with a given self-energy. For the derivation below we assume that the self-energy $\Sigma$ is local in space,

$$
\Sigma_{i j \sigma}\left(t, t^{\prime}\right)=\delta_{i j} \Sigma_{i \sigma}\left(t, t^{\prime}\right),
$$

which is true for DMFT and thus of wide range of applicability. The generalization of the formalism to nonlocal self-energies is briefly discussed in the conclusion.

In order to construct the auxiliary Hamiltonian $H_{\text {aux }}(t)$ we connect each individual site $i \equiv i_{0}$ of the crystal lattice to a set of additional sites $\mathcal{B}_{i}=\left\{i_{1}, i_{2}, i_{3}, \ldots\right\}$; see Fig. 1 . We will refer to $\mathcal{B}_{i}$ as the bath (but note that it is different from the bath of the effective single-site problem in DMFT). The additional dynamics between the bath and lattice sites are supposed to mimic the retardation effects of the self-energy $\Sigma$. We will see that this is achieved with an auxiliary Hamiltonian that has a quadratic form,

$$
\begin{aligned}
H_{\mathrm{aux}}(t)= & H_{0}(t)+\sum_{i \sigma} \sum_{l>0}\left(\epsilon_{i_{l} \sigma}(t)-\mu\right) a_{i_{l} \sigma}^{\dagger} a_{i_{l} \sigma} \\
& +\sum_{i \sigma} \sum_{l>0}\left(J_{i_{0} i_{l}}^{\sigma}(t) a_{i_{l} \sigma}^{\dagger} c_{i_{0} \sigma}+\text { H.c. }\right),
\end{aligned}
$$



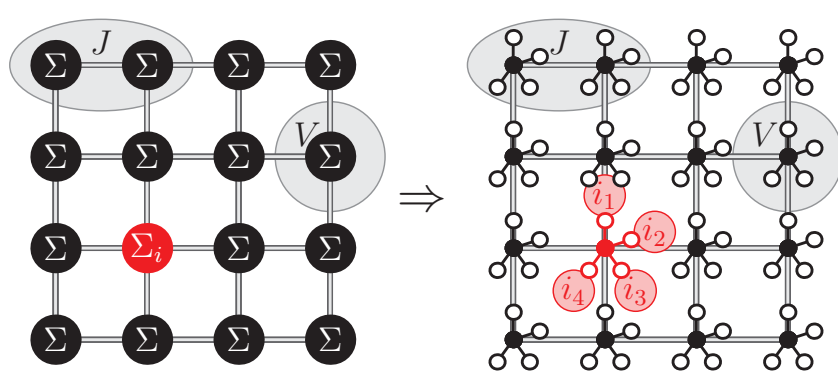

FIG. 1. (Color online) Under the assumption of a spatially local self-energy $\Sigma_{i}$ [compare with Eq. (6)], the lattice problem (1) can be mapped onto a noninteracting auxiliary system (right panel) where each lattice site $i$ is coupled to a set of bath orbitals $i_{1}, i_{2}, i_{3}$, etc. While the large dots in the left-hand panel indicate an on-site interaction $U$, the small dots in the right-hand panel refer to sites without a local Coulomb interaction; $J$ and $V$ denote the hopping and the external potential which remain the same in the auxiliary system; cf. Eq. (7).

where

$$
\begin{aligned}
H_{0}(t) & =\sum_{i j \sigma} J_{i j}(t) c_{i \sigma}^{\dagger} c_{j \sigma}+\sum_{i \sigma}\left[V_{i}(t)-\mu\right] n_{i \sigma} \\
& \equiv \sum_{i j \sigma}\left(h_{i j}(t)-\mu\right) c_{i \sigma}^{\dagger} c_{j \sigma}
\end{aligned}
$$

is the noninteracting part of Eq. (1), the operator $a_{i_{l} \sigma}^{\dagger}\left(a_{i_{l} \sigma}\right)$ creates (annihilates) an electron of spin $\sigma$ on the bath site $i_{l}$ for $l>0, \epsilon_{i_{l} \sigma}(t)$ are on-site energies of the bath orbitals, and $J_{i_{0} i_{l}}^{\sigma}(t)$ are the additional hopping matrix elements between site $i$ and bath orbitals $i_{l}$ which may depend on the spin.

The time-dependent parameters $J_{i_{0} i_{l}}^{\sigma}(t)$ and $\epsilon_{i_{l} \sigma}(t)$ must now be chosen such that the Green functions $G_{i_{0} j_{0} \sigma}^{\text {aux }}\left(t, t^{\prime}\right)$ of the noninteracting model defined by $H_{\text {aux }}(t)$ exactly equal the solution of the Dyson equation (2), i.e.,

$$
G_{i j \sigma}\left(t, t^{\prime}\right)=G_{i_{0} j_{0} \sigma}^{\mathrm{aux}}\left(t, t^{\prime}\right) .
$$

For this purpose, we consider the equations of motion for the Green functions $G_{i_{0} j_{0} \sigma}^{\text {aux }}\left(t, t^{\prime}\right)$,

$$
\begin{gathered}
{\left[i \partial_{t}+\mu\right] G_{i_{0} j_{0} \sigma}^{\mathrm{aux}}\left(t, t^{\prime}\right)-\sum_{r_{0}} h_{i_{0} r_{0}}(t) G_{r_{0} j_{0} \sigma}^{\mathrm{aux}}\left(t, t^{\prime}\right)} \\
=\delta_{i_{0} j_{0}} \delta_{\mathcal{C}}\left(t, t^{\prime}\right)+\sum_{l>0} J_{i_{0} i_{l}}^{\sigma}(t) G_{i_{l_{j} j_{0} \sigma}}^{\mathrm{aux}}\left(t, t^{\prime}\right),
\end{gathered}
$$

where $h_{r_{0} j_{0}}(t) \equiv h_{r j}(t)$ is defined by Eq. (8). Similarly, we can derive an equation of motion for the mixed bath-lattice term which enters the right-hand side of this equation $(l>0)$,

$$
\left[i \partial_{t}+\mu-\epsilon_{i_{l} \sigma}(t)\right] G_{i_{i} j_{0} \sigma}^{\mathrm{aux}}\left(t, t^{\prime}\right)=J_{i_{i} i_{0}}^{\sigma}(t) G_{i_{0} j_{0} \sigma}^{\mathrm{aux}}\left(t, t^{\prime}\right) .
$$

This equation can be solved by using the Green function $g\left(\epsilon_{i_{l} \sigma} ; t, t^{\prime}\right)$ for an isolated bath orbital with on-site energy $\epsilon_{i_{l} \sigma}$, which satisfies

$$
\left[i \partial_{t}+\mu-\epsilon_{i_{l} \sigma}(t)\right] g\left(\epsilon_{i_{l} \sigma} ; t, t^{\prime}\right)=\delta_{\mathcal{C}}\left(t, t^{\prime}\right),
$$

and has the explicit form

$$
g\left(\epsilon ; t, t^{\prime}\right)=i\left[f_{\beta}(\epsilon(0)-\mu)-\theta_{\mathcal{C}}\left(t, t^{\prime}\right)\right] e^{i \int_{t}^{t^{\prime}} d s[\epsilon(s)-\mu]} .
$$

Here, $f_{\beta}(\epsilon)=1 /\left(e^{\beta \epsilon}+1\right)$ denotes the Fermi-Dirac distribution, and $\theta_{\mathcal{C}}$ is the Heaviside step function on the contour. By convoluting Eq. (11) from the left with $g\left(\epsilon_{i_{l} \sigma} ; t, t^{\prime}\right)$ one obtains

$$
G_{i_{l} j_{0} \sigma}^{\text {aux }}\left(t, t^{\prime}\right)=\int_{\mathcal{C}} d s g\left(\epsilon_{i_{l} \sigma} ; t, s\right) J_{i_{l} i_{0}}^{\sigma}(s) G_{i_{0} j_{0} \sigma}^{\text {aux }}\left(s, t^{\prime}\right) .
$$

This result can be inserted into Eq. (10), which shows that $G_{i_{0} j_{0} \sigma}^{\text {aux }}\left(t, t^{\prime}\right)$ satisfies the equation of motion

$$
\begin{aligned}
& \sum_{r_{0}}\left[\delta_{i_{0} r_{0}}\left(i \partial_{t}+\mu\right)-h_{i_{0} r_{0}}(t)\right] G_{r_{0} j_{0} \sigma}^{\mathrm{aux}}\left(t, t^{\prime}\right) \\
& -\int_{\mathcal{C}} d s \Lambda_{i_{0} \sigma}^{\mathrm{aux}}(t, s) G_{i_{0} j_{0} \sigma}^{\mathrm{aux}}\left(s, t^{\prime}\right)=\delta_{i_{0} j_{0}} \delta_{\mathcal{C}}\left(t, t^{\prime}\right),
\end{aligned}
$$

with

$$
\Lambda_{i_{0} \sigma}^{\mathrm{aux}}\left(t, t^{\prime}\right)=\sum_{l>0} J_{i_{0} i_{l}}^{\sigma}(t) g\left(\epsilon_{i_{l} \sigma} ; t, t^{\prime}\right) J_{i_{i} i_{0}}^{\sigma}\left(t^{\prime}\right) .
$$

By comparing Eq. (15) with the differential form (5) of the Dyson equation (2), we see that the relation (9) is satisfied, provided we can find parameters $J_{i_{0} i_{l}}^{\sigma}(t)$ and $\epsilon_{i_{l} \sigma}(t)$ such that

$$
\Lambda_{i_{0} \sigma}^{\mathrm{aux}}\left(t, t^{\prime}\right)=\Sigma_{i \sigma}\left(t, t^{\prime}\right)
$$

for all times $t$ and $t^{\prime}$ located on the time contour $\mathcal{C}$. We note that condition (17) must hold only for contributions of the self-energy which are beyond the mean-field level while any Hartree contribution can be absorbed in an effective potential,

$$
V_{i \sigma}(t)=V_{i}(t)+U(t)\left(\left\langle n_{i \bar{\sigma}}\right\rangle-\frac{1}{2}\right) .
$$

With Eqs. (16) and (17), the problem of determining the parameters of each independent bath $\mathcal{B}_{i}$ becomes identical to that of representing a nonequilibrium DMFT action by a single-impurity Anderson model; see [24]. The only difference is that instead of the hybridization function of the DMFT bath we here fit the self-energy. In [24], the existence of solutions and an explicit construction of a solution has been discussed.

A short way of summarizing the derivation along the lines of Eqs. (11)-(15) is to say that the effective action obtained from the auxiliary model (by integrating out the bath sites) is given by

$$
S_{\text {aux }}=S_{0}-i \sum_{i_{0} \sigma} \int_{\mathcal{C}} d t \int_{\mathcal{C}} d t^{\prime} \Lambda_{i_{0} \sigma}^{\mathrm{aux}}\left(t, t^{\prime}\right) c_{i_{0} \sigma}^{\dagger}(t) c_{i_{0} \sigma}\left(t^{\prime}\right),
$$

where $S_{0}=-i \int_{\mathcal{C}} d t H_{0}(t)$ [24]. The single-particle Green functions of this quadratic action satisfy the Dyson equation (2), provided that Eq. (17) is satisfied.

\section{Application to time-dependent Hubbard I}

In this section we illustrate the approach within the Hubbard I approximation, for which the representation (17) of the selfenergy can be derived analytically. Within the (nonvariational) Hubbard-I approximation, the self-energy of the lattice is approximated by the self-energy of an isolated Hubbard site with Hamiltonian $H_{\text {at }}(t)=U(t) n_{\uparrow} n_{\downarrow}+\sum_{\sigma} \epsilon_{\sigma} n_{\sigma}$. The approximation is the simplest variant of the nonequilibrium cluster perturbation theory [17], in which the self-energy is computed from a small cluster of the lattice.

For simplicity we consider the case in which the model is driven out of equilibrium only by external fields, while 
the Hubbard interaction is time independent. The Hubbard-I self-energy can then be computed from an isolated site in equilibrium. The corresponding Matsubara Green function $G_{\sigma}^{\text {at }}\left(i \omega_{n}\right)$ for the Hamiltonian $H_{\text {at }}$ is given by

$$
G_{\sigma}^{\mathrm{at}}\left(i \omega_{n}\right)=\frac{1-\left\langle n_{\bar{\sigma}}\right\rangle_{\mathrm{at}}}{i \omega_{n}-\epsilon_{\sigma}}+\frac{\left\langle n_{\bar{\sigma}}\right\rangle_{\mathrm{at}}}{i \omega_{n}-U-\epsilon_{\sigma}},
$$

and the self-energy is obtained from inverting $G_{\sigma}^{\text {at }}\left(i \omega_{n}\right)=$ $\left[i \omega_{n}-\epsilon_{\sigma}-\Sigma_{\sigma}^{\mathrm{at}}\left(i \omega_{n}\right)\right]^{-1}$. We find

$$
\Sigma_{\sigma}^{\mathrm{at}}\left(i \omega_{n}\right)=U\left\langle n_{\bar{\sigma}}\right\rangle_{\mathrm{at}}+\frac{a_{\sigma}^{2}}{i \omega_{n}-E_{\sigma}},
$$

with

$$
a_{\sigma}^{2}=U^{2}\left\langle n_{\bar{\sigma}}\right\rangle_{\text {at }}\left\langle 1-n_{\bar{\sigma}}\right\rangle_{\text {at }}, \quad E_{\sigma}=U\left\langle 1-n_{\bar{\sigma}}\right\rangle_{\text {at }}+\epsilon_{\sigma} .
$$

The analytical continuation of $\Sigma_{\sigma}^{\mathrm{at}}\left(i \omega_{n}\right)$ to the Keldysh contour gives

$$
\Sigma_{\sigma}^{\mathrm{at}}\left(t, t^{\prime}\right)=U\left\langle n_{\bar{\sigma}}\right\rangle_{\mathrm{at}} \delta_{\mathcal{C}}\left(t, t^{\prime}\right)+a_{\sigma}^{2} g\left(E_{\sigma} ; t, t^{\prime}\right),
$$

where $g\left(E_{\sigma} ; t, t^{\prime}\right)$ is given by Eq. (13).

The time-nonlocal part of the self-energy (23) is precisely of the form (16). As a result, solving the Dyson equation with the self-energy $\Sigma_{\sigma}^{\text {at }}\left(t, t^{\prime}\right)$ at each lattice site is equivalent to solving the noninteracting lattice problem with only one additional bath orbital per lattice site $i$ which is characterized by an on-site energy $\epsilon_{i_{1}}^{\sigma}=E_{\sigma}$ and a time-independent hopping $J_{i_{0} i_{1}}^{\sigma}=a_{\sigma}$. The numerical solution of this single-particle problem involves no memory integrals, and it can thus be carried out to arbitrarily large times without any restriction on the memory. A similar exact representation of the self-energy with finitely many bath orbitals is possible in general when the (time-dependent) Lehmann representation of $\Sigma$ has finitely many terms. This might be useful for certain applications of nonequilibrium cluster perturbation theory with small clusters.

\section{Decomposition of the self-energy}

In general, the representation of the self-energy defined by Eqs. (16) and (17) is not known analytically. To solve Eqs. (16) and (17) for the bath parameters, we separately consider the various analytical components of the self-energy. In general, each contour function can be parametrized in terms of five components according to different locations of the time arguments on $\mathcal{C}$. For the one-particle Green function, we have exemplarily

$$
\begin{aligned}
G_{i j \sigma}^{<}\left(t, t^{\prime}\right) & =i\left\langle c_{j \sigma}^{\dagger}\left(t^{\prime}\right) c_{i \sigma}(t)\right\rangle, \\
G_{i j \sigma}^{>}\left(t^{\prime}, t\right) & =-i\left\langle c_{i \sigma}\left(t^{\prime}\right) c_{j \sigma}^{\dagger}(t)\right\rangle, \\
G_{i j \sigma}^{-}(t, \tau) & =-i\left\langle c_{j \sigma}^{\dagger}(\tau) c_{i \sigma}(t)\right\rangle, \\
G_{i j \sigma}^{-}(\tau, t) & =-i\left\langle c_{i \sigma}(\tau) c_{j \sigma}^{\dagger}(t)\right\rangle, \\
G_{i j \sigma}^{\mathrm{M}}(\tau) & =-\left\langle c_{i \sigma}(\tau) c_{j \sigma}^{\dagger}(0)\right\rangle,
\end{aligned}
$$

where the argument $t\left(t^{\prime}\right)$ is here situated on the upper (lower) real branch of the contour and $\tau$ refers to a time on the imaginary track. In addition, we have the Hermitian symmetry relations

$$
\begin{aligned}
& X_{i j \sigma}^{\gtrless}\left(t, t^{\prime}\right)=-\left[X_{j i \sigma}^{\gtrless}\left(t^{\prime}, t\right)\right]^{*}, \\
& X_{i j \sigma}^{\neg}(t, \tau)=X_{j i \sigma}^{-}(\beta-\tau, t)^{*}
\end{aligned}
$$

for the components of the Green function $(X=G)$ and the self-energy $(X=\Sigma)$.

While the construction of bath parameters for arbitrary initial states is discussed in detail in [24], we start in Secs. III and IV from an uncorrelated initial state, i.e., $U(t)=0$ for times $t \leqslant 0$. In this case, the Matsubara and mixed components of the self-energy vanish, $\Sigma^{\mathrm{M}}=\Sigma^{-}=\Sigma^{\urcorner}=0$, and the remaining components of the self-energy are the lesser and greater functions $\Sigma^{<}$and $\Sigma^{>}$which have real time arguments. Following [24], we can fit them separately by taking the energies of the bath sites entering Eq. (13) to be time independent, i.e., $\epsilon_{i l}(t)=\mu$ for $t>0$, and by choosing the initial energies $\epsilon_{i_{l} \sigma}(0)$ such that $f\left(\epsilon_{i_{l} \sigma}(0)-\mu\right)$ is either 0 or 1 . This leads to a representation of the self-energy with two sets of bath orbitals, $\mathcal{B}_{i}^{<}$and $\mathcal{B}_{i}^{>}$, where all sites in $\mathcal{B}_{i}^{<}$ $\left(\mathcal{B}_{i}^{>}\right)$are initially occupied (empty) and $\mathcal{B}_{i}=\mathcal{B}_{i}^{<} \cup \mathcal{B}_{i}^{>}$. More precisely, we have

$$
-i \Sigma_{i \sigma}^{<}\left(t, t^{\prime}\right)=\sum_{l \in \mathcal{B}_{i}^{<}} J_{i_{0} i_{l}}^{\sigma}(t)\left[J_{i_{l} i_{0}}^{\sigma}\left(t^{\prime}\right)\right]^{*},
$$

and

$$
i \Sigma_{i \sigma}^{>}\left(t, t^{\prime}\right)=\sum_{l \in \mathcal{B}_{i}^{>}} J_{i_{0} i_{l}}^{\sigma}(t)\left[J_{i_{l} i_{0}}^{\sigma}\left(t^{\prime}\right)\right]^{*} .
$$

In the case of particle-hole symmetry, i.e., for $\mu=0$ in Eq. (1), one of the two equations is redundant because the greater and lesser functions are then related through $\Sigma_{i \sigma}^{<}\left(t, t^{\prime}\right)=$ $\Sigma_{i \sigma}^{>}\left(t, t^{\prime}\right)^{*}$. If we discretize the times $t$ and $t^{\prime}$ according to $t=t_{n}=n \Delta t$ and $t^{\prime}=t_{n^{\prime}}=n^{\prime} \Delta t$ with $n, n^{\prime} \in\{0,1,2, \ldots, N\}$, Eqs. (26) and (27) have the form of standard matrix decompositions. Thus we can obtain an exact representation of $\Sigma_{i \sigma}$ on the given time mesh using in total $L_{i}=2(N+1)$ bath orbitals.

More interesting is the possibility to find an approximate but still accurate representation using fewer bath orbitals by applying a suitable low-rank approximation to Eqs. (26) and (27),

$$
\left(-i \Sigma_{i \sigma}^{<}\right)_{n n^{\prime}} \approx \sum_{l=1}^{L_{i}^{<}} J_{i_{0} i_{l}}^{\sigma}\left(t_{n}\right)\left[J_{i_{0} i_{l}}^{\sigma}\left(t_{n^{\prime}}\right)\right]^{*},
$$

where $L_{i}^{<}$is a fixed finite number of bath sites which is smaller than the number of time steps $N$ [similarly for $\left.\left(i \Sigma_{i \sigma}^{>}\right)\right]$. In the following, we will apply the low-rank Cholesky decomposition to (26) and (27) in order to obtain the hopping parameters $J_{i_{0} i_{l}}^{\sigma}(t)$ on the discretized time mesh $t=t_{n}$, which has the advantage of being causal, i.e., the parameters at time $t=$ $m \Delta t$ only depend on the values $\left( \pm i \Sigma_{i \sigma}^{\gtrless}\right)_{n n^{\prime}}$ with $n, n^{\prime} \leqslant m$. For technical details concerning the low-rank approximation (28) we refer the reader to [24].

If $L_{i}^{\gtrless} \ll N$, Eq. (28) enables a very compact representation of the self-energy where instead of $(N+1)^{2}$ elements per component $\Sigma_{i \sigma}$ only a small number of $L_{i}(N+1)$ elements, namely $L_{i}=L_{i}^{>}+L_{i}^{<}$hopping matrix elements for $N+1$ times, are required to define the time dependence of the 
self-energy. In practice, the numbers $L_{i}^{>}$and $L_{i}^{<}$act as convergence parameters, and their minimum value depends on the maximum evolution time, cf. Sec. III.

\section{E. Propagation schemes}

Since the auxiliary model (7) is a noninteracting problem, Green functions can be determined by closed equations of motion, cf. (10). In short, we may write

$$
\begin{aligned}
\left\{i \partial_{t}+\mu-h_{\mathrm{aux}}^{\sigma}(t)\right\} G_{\sigma}^{\mathrm{aux}}\left(t, t^{\prime}\right) & =\delta_{\mathcal{C}}\left(t, t^{\prime}\right), \\
\left\{-i \partial_{t^{\prime}}+\mu-h_{\mathrm{aux}}^{\sigma}\left(t^{\prime}\right)\right\} G_{\sigma}^{\mathrm{aux}}\left(t, t^{\prime}\right) & =\delta_{\mathcal{C}}\left(t, t^{\prime}\right),
\end{aligned}
$$

where $h_{\text {aux }}^{\sigma}(t)$ is the single-particle Hamiltonian of the auxiliary problem, and all quantities are viewed as matrices with space and bath orbital indices. If we label the sites of the crystal lattice with $i=0,1,2,3$, etc. and let $L=L_{i}^{<}+L_{i}^{>}$denote the number of bath orbitals attached to each lattice site (for notational simplicity we assume all local self-energies to be represented with the same number of bath orbitals), we can cast the single-particle Hamiltonian $h_{\text {aux }}^{\sigma}(t)$ into the following block matrix form (time arguments are omitted),

$$
h_{\text {aux }}^{\sigma}(t)=\left(\begin{array}{cccc}
a_{00 \sigma} & b_{01} & b_{02} & \ldots \\
b_{10} & a_{11 \sigma} & b_{12} & \ldots \\
b_{20} & b_{21} & a_{22 \sigma} & \ddots \\
\vdots & \vdots & \ddots & \ddots
\end{array}\right)(t),
$$

where all $a$ and $b$ blocks are of dimension $(L+1) \times(L+1)$. While the $a$ blocks in Eq. (30) include the hopping to the bath and the effective potential $V_{i \sigma}(t)$, the $b$ blocks involve the hopping terms which connect different lattice sites, i.e., (note that by definition $i_{0}=i$ and $j_{0}=j$ ),

$$
\begin{gathered}
a_{i i \sigma}(t)=\left(\begin{array}{cccc}
V_{i_{0} \sigma}(t) & J_{i_{0} i_{1}}^{\sigma}(t) & \ldots & J_{i_{0} i_{L}}^{\sigma}(t) \\
J_{i_{1} i_{0}}^{\sigma}(t) & 0 & \ldots & 0 \\
\vdots & \vdots & \ddots & \vdots \\
J_{i_{L} i_{0}}^{\sigma}(t) & 0 & \ldots & 0
\end{array}\right), \\
b_{i j}(t)=\left(\begin{array}{cccc}
J_{i_{0} j_{0}}(t) & 0 & \ldots & 0 \\
0 & 0 & \ldots & 0 \\
\vdots & \vdots & \ddots & \vdots \\
0 & 0 & \ldots & 0
\end{array}\right)
\end{gathered}
$$

Note that in the case of nearest-neighbor hopping most of the entries in the off-diagonal blocks $b$ vanish, and the Hamiltonian $h_{\text {aux }}^{\sigma}$ becomes extremely sparse.

If the initial state at time $t=0$ is described by the one-particle density matrix $\rho_{i_{l} i_{k} \sigma}^{\text {aux }}(0)=\left\langle c_{i_{k} \sigma}^{\dagger}(0) c_{i_{l} \sigma}(0)\right\rangle_{\text {aux }}$, the solution of Eqs. (29a) and (29b) for the lesser and greater components of the auxiliary Green function gives

$$
\left[G_{\sigma}^{\mathrm{aux}}\right]^{\gtrless}\left(t, t^{\prime}\right)=\mp i U_{\sigma}(t, 0) R_{\sigma}^{\gtrless} U_{\sigma}^{\dagger}\left(t^{\prime}, 0\right),
$$

where $R_{\sigma}^{>}=1-\rho_{\sigma}^{\text {aux }}(0), R_{\sigma}^{<}=\rho_{\sigma}^{\text {aux }}(0)$, and

$$
U_{\sigma}\left(t^{\prime}, t\right)=T_{\mathrm{t}} \exp \left(-i \int_{t}^{t^{\prime}} d s h_{\text {aux }}^{\sigma}(s)\right)
$$

is the single-particle propagator. In Appendix A, the time propagation is explained in detail.

In general, the self-energy is a functional of the Green function (e.g., through the DMFT self-consistency and the solution of the impurity problem, or through a self-consistent diagrammatic expansion). In Eqs. (29a) and (29b), the selfconsistency condition is rather hidden in the dependence of the one-particle Hamiltonian $h_{\text {aux }}^{\sigma}=h_{\text {aux }}^{\sigma}\left[G^{\text {aux }}, \Sigma\right]$ on the auxiliary Green function $G^{\text {aux }}$ [through the Hartree contribution, Eq. (18)], and the time nonlocal part of the self-energy $\Sigma$. In principle, there are two possibilities to obtain self-consistent solutions. On the one hand, we can determine the auxiliary Green function for a fixed self-energy for all times and then iterate Eqs. (29a) and (29b) by updating the self-energy [and in turn $\left.h_{\sigma}^{\mathrm{aux}}(t)\right]$ on the whole time mesh. This is easy to implement, but can require a large number of iterations. On the other hand, we can directly exploit the causality of the Cholesky decomposition of the self-energy [recall discussion below Eq. (28)] and set up a time propagation scheme where the self-consistency is established on each time slice $n$ separately [24]. In combination with an appropriate (typically higher-than-linear order) extrapolation of the hopping matrix elements $J_{i_{0} i_{l}}^{\sigma}(t)$ for times $t \leqslant t_{n}$ onto the subsequent time slice $n+1$, this guarantees a small number of local iterations which is very advantageous. In Appendix B, we describe how one further can apply the Krylov method [39] to evaluate the action of the unitary time evolution operator $U_{\sigma}\left(t^{\prime}, t\right)$ in Eq. (33) and how the time stepping algorithm is straightforwardly parallelized.

Finally, we mention that the auxiliary bath approach is beneficial also in terms of memory consumption. Usually, the numerical solution of the Kadanoff-Baym equations is limited by the available computer memory because the total Green function $G_{i j \sigma}\left(t, t^{\prime}\right)$ is stored in order to evaluate the memory kernel on each time slice [32]. Long simulations with many orbital degrees of freedom require for this reason massive parallelization and a suitable distribution of memory over several compute nodes, e.g., [22,34]. In the auxiliary bath formalism, on the contrary, it is sufficient to store the parameters of the local Hamiltonian (30). The number of nonzero parameters is thus determined by the memory needed to store the self-energy $\Sigma_{i \sigma}\left(t, t^{\prime}\right)$ which requires by definition considerably less memory than the Green function if $\Sigma$ is sufficiently local in space, and if it can be represented accurately within a suitable low-rank approximation, cf. Eq. (28).

\section{SCALING BEHAVIOR}

In the following, we test the low-rank decomposition of the self-energy [Eq. (28)] and illustrate the time propagation of the auxiliary system for two simple cases. We will first analyze how well a given self-energy $\Sigma\left(t, t^{\prime}\right)$ on a time window $t, t^{\prime} \leqslant t_{\max }$ can be represented with a fixed number $L$ of bath orbitals. Subsequently, we will assess how the solution of the resulting auxiliary problem converges against the full solution of the Dyson equation with increasing $L$.

To analyze these questions we will use a test self-energy that is generated by solving the Hubbard model (1) on a small cluster within self-consistent second-order perturbation theory, 
(a) $U_{\mathrm{f}}=1.0$
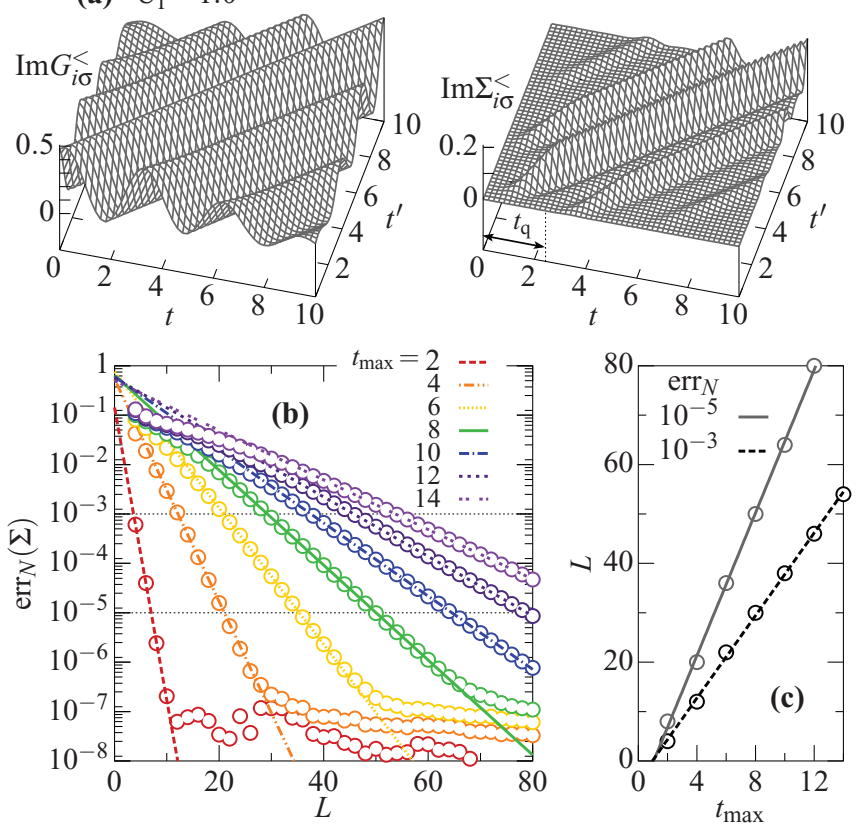

FIG. 2. (Color online) (a) Self-consistent Green function $G_{i \sigma}^{<}$and local self-energy $\Sigma_{i \sigma}^{<}$of the $2 \times 2$ cluster as obtained in second Born approximation from the Dyson equation (2). The parameters are $\beta=10, \mu=0, J=J_{0}, t_{\mathrm{q}}=2.5$, and $U_{\mathrm{f}}=1.0$. (b) Error of the approximate self-energy evaluated with Eq. (28) for various lengths $t_{\max }$ of the time evolution and different sizes $L$ of the bath. (c) Dependence of the accessible maximum time $t_{\max }$ on $L$ for a given maximum permitted error $\operatorname{err}_{N}(\Sigma)$ in the self-energy.

i.e., within the second Born approximation. To be precise, the time nonlocal part of the self-energy is taken to be

$$
\Sigma_{i \sigma}^{\gtrless}\left(t, t^{\prime}\right)=U(t) U\left(t^{\prime}\right)\left[G_{i \sigma}^{\gtrless}\left(t, t^{\prime}\right)\right]^{2} G_{i \sigma}^{\lessgtr}\left(t^{\prime}, t\right),
$$

where $G$ are the self-consistent solutions of the Dyson equation (2). We solve Eqs. (35) and (2) either for a single isolated lattice site or for a cluster of $2 \times 2$ lattice sites with time-independent nearest-neighbor hopping $J_{0}$. To drive the system out of equilibrium, we modify the interaction as a function of time,

$$
U(t)=U_{\mathrm{f}} \times\left\{\begin{array}{cc}
\frac{1}{2}\left[1-\cos \left(\pi t / t_{\mathrm{q}}\right)\right], & t \leqslant t_{\mathrm{q}}, \\
1, & t>t_{\mathrm{q}},
\end{array}\right.
$$

with ramp time $t_{\mathrm{q}}=2.5$, starting from an uncorrelated state at temperature $\beta=10$ and half filling $(\mu=0)$. In Figs. 2(a) and 3(a), we show self-consistent reference data for the Green functions and the self-energies for the single-site and four-site cluster, respectively. Below, all energies (times) are measured in units of the (inverse) hopping $J_{0}\left(J_{0}^{-1}\right)$.

Note that we use second-order perturbation theory as an easy way to generate a self-energy with the correct analytical properties and a functional form that is similar to selfenergies obtained within DMFT for large systems: $\Sigma^{<}\left(t, t^{\prime}\right)$ and $\Sigma^{>}\left(t, t^{\prime}\right)$ fall off as a function of $t-t^{\prime}$, but have both nontrivial structure as a function of average time $\left(t+t^{\prime}\right) / 2$ (due to the interaction ramp) and as a function of $t-t^{\prime}$; see Figs. 2(a) and 3(a). The true self-energy of a single-site cluster
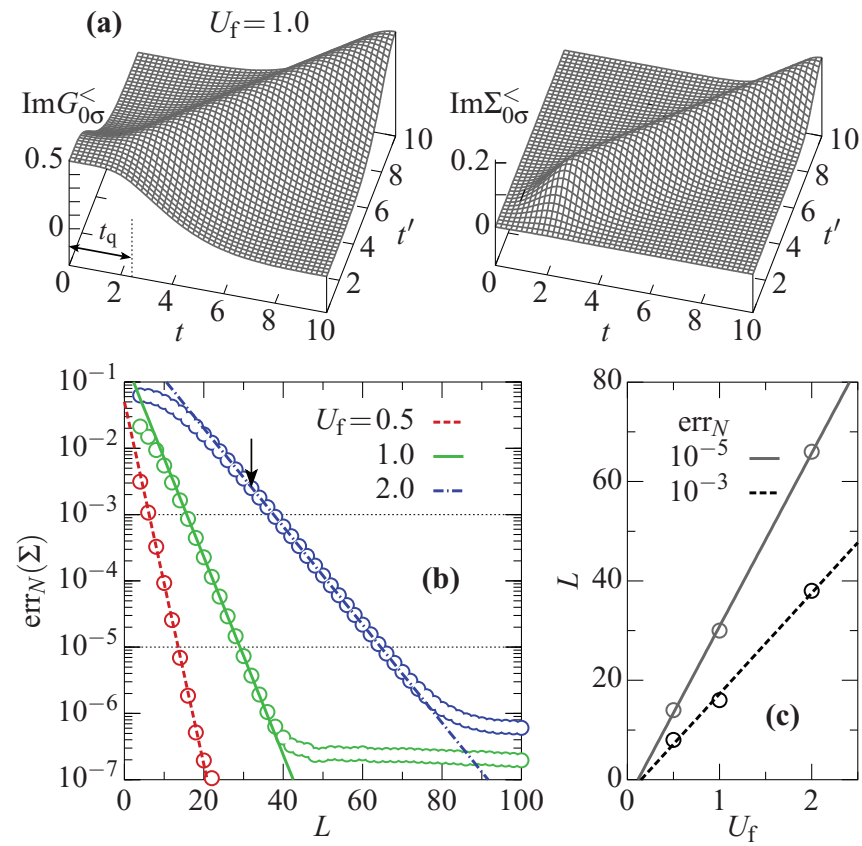

FIG. 3. (Color online) (a) Time evolution of the lesser Green function and the lesser self-energy as obtained from Eq. (2) in second Born approximation for $U_{\mathrm{f}}=1.0, t_{\mathrm{q}}=2.5, \beta=10$, and $\mu=0$. (b) Low-rank Cholesky decomposition of the test self-energy in the interval $\left[0, t_{\max }\right]=[0,10]$ for different parameters $U_{\mathrm{f}}$. Displayed is the error $\operatorname{err}_{N}(\Sigma)$ as defined in Eq. (37) with $N=400$ time steps as function of the number of bath orbitals $L$ used in Eq. (28). (c) Scaling of the number of bath sites $L$ with $U_{\mathrm{f}}$ for fixed errors $\operatorname{err}_{N}(\Sigma)$.

is of course different and not well described by second-order perturbation theory.

\section{A. Representation of the self-energy}

To analyze the representation of the self-energy, we compare the given input self-energy to the low-rank approximation $\Sigma_{\text {approx }}$ which is obtained from the matrix decomposition (28). For the time discretization introduced in Sec. IID (i.e., $t_{n}=$ $n \Delta t$ and $t_{n^{\prime}}=n^{\prime} \Delta t$ with $\left.n, n^{\prime} \in\{0,1,2, \ldots, N\}\right)$, we define the corresponding error as

$$
\operatorname{err}_{N}(\Sigma)=\sum_{n, n^{\prime}} \sum_{\alpha \in\{>,<\}} \frac{\left|\Sigma^{\alpha}\left(t_{n}, t_{n^{\prime}}\right)-\Sigma_{\text {approx }}^{\alpha}\left(t_{n}, t_{n^{\prime}}\right)\right|}{2(N+1)^{2}} .
$$

Figure 2(a) displays the input Green function and selfenergy for $U_{\mathrm{f}}=1.0$ for the self-energy of the four-site cluster. In Fig. 2(b), we plot the error $\operatorname{err}_{N}(\Sigma)$ of the self-energy decomposition (28) as a function of the number of bath orbitals $L$ for different lengths $t_{\max }$ of the time propagation. The size of the time step is thereby fixed to $\Delta t=0.025$, such that $N=80$ for $t_{\max }=2$ and $N=560$ for $t_{\max }=14$. Independent of the value of $t_{\max }$, we find an exponentially small error for a sufficiently large number of bath sites [the plateaus for $\operatorname{err}_{N}(\Sigma)<10^{-6}$ can be attributed to a small number $\lambda>0$ (typically $\lambda=10^{-8}$ ) which we add to the diagonal matrix elements $\Sigma(t, t)$ of the self-energy in order to guarantee that the matrices $\left(-i \Sigma^{<}\right)$and $\left(i \Sigma^{>}\right)$are positive definite]. On the other hand, we observe that an accordingly larger bath is required 
in order to reach longer simulation times with the same global error $\operatorname{err}_{N}(\Sigma)$.

The maximum time which is accessible under a fixed error $\operatorname{err}_{N}(\Sigma)$ depends linearly on the number of bath orbitals, see Fig. 2(c). Quantitatively, we find that it is sufficient to choose $L$ considerably smaller than the total number of time steps $N$. For example, with 40 bath sites at $t_{\max }=10$, an error less than $10^{-3}$ is achieved. On the other hand, $L=40$ bath sites correspond to an effective time step size of $\Delta t=10 / 40=$ 0.25 which would be too large to obtain numerically converged results in a solution of the integral equation (2). Hence the lowrank decomposition has allowed us to effectively compress the information stored in the self-energy $\Sigma\left(t, t^{\prime}\right)$.

The quality of the representation depends on the functional form of the self-energy. We can study this dependence systematically for the test self-energy obtained for the isolated site, which has a particularly simple shape: It is characterized by a monotonous decay as a function of the difference time $t-t^{\prime}$ [see Fig. 3(a)], where the decay time decreases with increasing $U_{\mathrm{f}}$. Figure $3(\mathrm{~b})$ shows the $\operatorname{error} \operatorname{err}_{N}(\Sigma)$ for different values of $U_{\mathrm{f}}$ as a function of the total number of bath sites $L$ used in the low-rank Cholesky decomposition. As observed for the four-site self-energy, the error decreases exponentially with increasing number of bath sites, and $L$ can be chosen smaller than the number of time steps. However, the representation of the self-energy for larger $U_{\mathrm{f}}$ requires a larger bath to reach the same level of accuracy. Figure 3(c) indicates a linear scaling between the size of the bath and the strength of the Coulomb interaction for a given maximum error. Taking into account the functional form of $\Sigma$, this indicates that the representation of a self-energy which is localized close to the time diagonal needs more bath orbitals, which can be understood at least qualitatively: If the self-energy decays to zero for $\left|t-t^{\prime}\right|$ larger than some "memory time" $t_{\mathrm{c}}$, this can be incorporated into the representation (28) if each bath site is coupled at most for a time period $2 t_{\mathrm{c}}$. Thereafter, new bath sites must be coupled to the system.

\section{B. Solution of the Dyson equation with a low-rank approximation}

As the next step, we demonstrate that the auxiliary bath formalism is able to reproduce the same Green function $G_{i j \sigma}\left(t, t^{\prime}\right)$ as the direct solution of Eq. (2). To this end, we propagate the auxiliary system (7) in time and, following the scheme described in Sec. II E, extract the self-consistent Green function for different but fixed sizes of the bath. Figure 4 shows the results for $L=4,8,16$, and 32 bath orbitals and $U_{\mathrm{f}}=2.0$ for the single-site cluster (again we use $N=400$ time steps). If the size of the bath is too small, we observe that the time evolution of the Green function develops artifacts in form of additional oscillations as function of $t$ and $t^{\prime}$. For larger values of $L$, these artifacts shift to later times and finally disappear, such that the exact solution is well recovered to longer and longer times. The Green function for $L=32$ is (by eye) barely distinguishable from the exact one. This is consistent with an error of $\operatorname{err}_{N}(\Sigma)<10^{-2}$ which we find for the self-energy in Fig. 3(b); see the black arrow.

The convergence of the low-rank approximation with the number of bath sites can be seen even more directly from
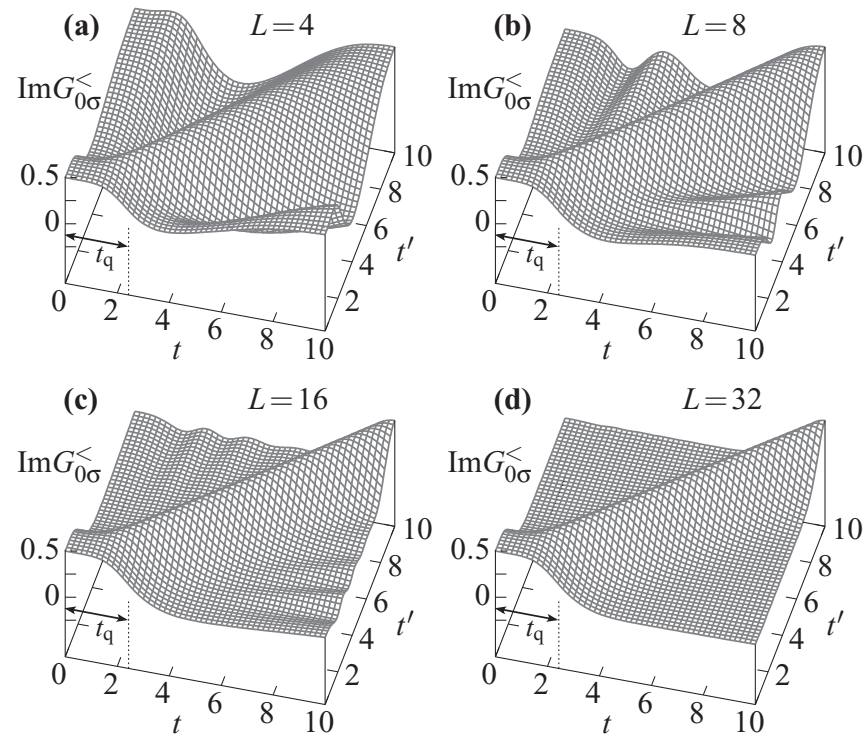

FIG. 4. Fully self-consistent results for the imaginary part of the Green function $G_{0 \sigma}^{<}\left(t, t^{\prime}\right)$ for the single-site cluster as obtained in second Born approximation from the time propagation of the auxiliary system for different numbers of bath orbitals $L$. The interaction strength for times $t \geqslant t_{\mathrm{q}}$ is $U_{\mathrm{f}}=2.0$. All other parameters are as in Fig. 3, in particular $t_{\max }=10$ and $N=400$.

the time evolution of single-time observables. In Fig. 5, we exemplarily show results for the local double occupation in the four-site cluster for different $L$. The double occupation, which is proportional to the interaction energy, is obtained from the convolution

$$
\begin{aligned}
\langle d\rangle(t) & =\sum_{i}\left\langle d_{i}\right\rangle(t) \\
& =-\frac{i}{U(t)} \sum_{i}\left\{\int_{\mathcal{C}} d s \Sigma_{i \sigma}(t, s) G_{i_{0} i_{0} \sigma}^{\mathrm{aux}}\left(s, t^{\prime}\right)\right\}_{t^{\prime}=t^{+}} .
\end{aligned}
$$

The maximum time up to which the solution is converged increases with the number of bath orbitals. As observed for the representation of the self-energy, the number $L$ of bath sites required to reach a given accuracy is smaller than the number of time slices needed in the conventional solution of the Dyson equation.

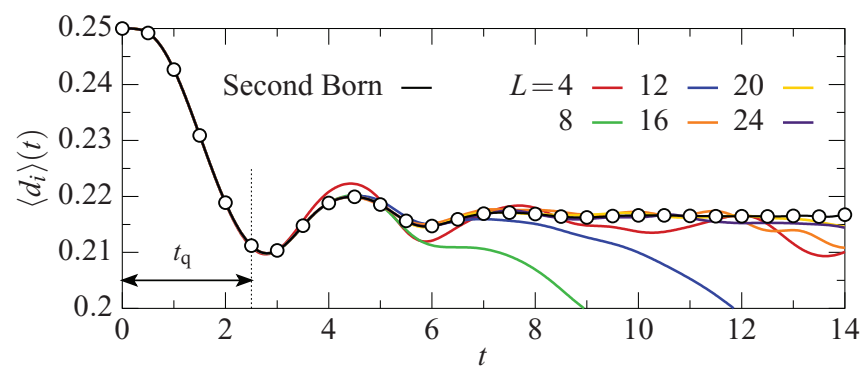

FIG. 5. (Color online) Time evolution of the local double occupation $\left\langle d_{i}\right\rangle(t)$ in the four-site cluster for $U_{\mathrm{f}}=1.0$ in the second-order Born approximation (black open dots and black solid line). The colored curves show the results for different sizes of the bath. 


\section{2D OPTICAL LATTICE IN A HARMONIC TRAP}

In this section, we apply the auxiliary Hamiltonian approach to investigate the interaction quench in the Hubbard model. We particularly focus on the effect of the confinement potential, which is present for experiments with ultracold atoms. Interaction quenches in Bose- and Fermi-Hubbard models have been extensively studied in homogeneous systems [8,10,11,35,36]. After a quench from $U=0$ to the weakly interacting regime, the system rapidly evolves to a state in which kinetic energy and potential energy are almost thermalized, while the momentum distribution function $n(\boldsymbol{k})$ is still far from its final value. In this prethermalized state [9] rapid thermalization is inhibited by an infinite number of almost conserved quantities which exist due to the vicinity of the noninteracting state [37]. Thermalization at longer times and weak coupling is then captured by kinetic equations $[7,8]$.

Typically, the nonthermal nature of the intermediate state is most clearly evidenced by a discontinuity of $n(\boldsymbol{k})$ across the Fermi surface, which would be absent at any temperature $T>0[8,10,11]$. In the presence of a confinement potential, however, sharp features like the discontinuity in the momentum occupation are expected to be blurred, and, moreover, the interaction quench in a trap might excite collective density oscillations of the atom cloud (e.g., a breathing mode), which are superimposed to the relaxation dynamics. The possible observation of prethermalization in experiment thus requires a good understanding of effects caused by the trapping potential. Below, we will investigate signatures of a two-stage relaxation for a system with a rather narrow confinement, where density oscillations after the quench become very pronounced.

\section{A. Setup}

We study the Hubbard model (1) with nearest-neighbor hopping $J_{i j}=\delta_{\langle i j\rangle} J_{0}$ on a square lattice with $10 \times 10$ sites. The optical trap is modeled by a parabolic confinement potential $V_{i}$ characterized by two frequencies, $\omega_{1}$ and $\omega_{2}$,

$$
V_{i}(t)=\omega_{1}^{2}\left(\mathbf{R}_{i} \hat{\mathbf{a}}_{1}\right)^{2}+\omega_{2}^{2}\left(\mathbf{R}_{i} \hat{\mathbf{a}}_{2}\right)^{2} .
$$

Here, $\hat{\mathbf{a}}_{j}$ are the unit vectors along the principle axes of the trap, and the vector $\mathbf{R}_{i}$ is pointing from the trap center to the lattice site $i$ (the lattice spacing is set to 1). In the following we compare results for a rotationally symmetric trap with $\omega_{1}^{2}=$ $\omega_{2}^{2}=0.5 J_{0}$ [referred to as system A; see Fig. 6(a)] with those for an elongated trap with $\omega_{1}^{2}=0.5 J_{0}$ and $\omega_{2}^{2}=J_{0}$ which is rotated by $30^{\circ}$ with respect to the lattice [system B; Fig. 6(b)]. The inverse temperature is $\beta=10$, and we fix the average particle number in the trap to $\langle N\rangle=\left\langle N_{\uparrow}\right\rangle+\left\langle N_{\downarrow}\right\rangle=40$ by tuning the chemical potential $\mu$ of the initial state. The hopping $J_{0}$ and the inverse hopping $J_{0}^{-1}$ define the units for energy and time, respectively. The system is excited by an almost sudden ramp of the electron-electron interaction starting from the noninteracting state. The time dependence of the quench follows Eq. (36) with $t_{\mathrm{q}}=0.5$. In all calculations we use the DMFT approximation and evaluate the local self-energy in the second-order Born approximation [cf. Eq. (35)].

Before discussing the results, it is interesting to look at the reduction in computational resource requirements achieved by the auxiliary bath scheme for the current problem. For

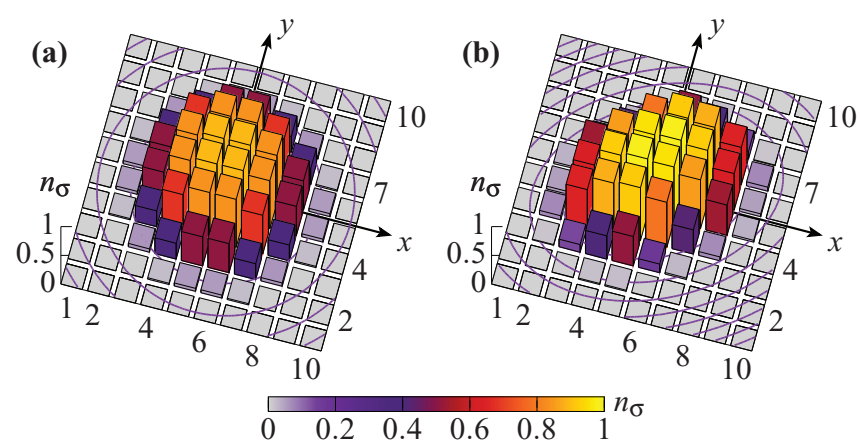

FIG. 6. (Color online) Density profiles $n_{\sigma}\left(\mathbf{R}_{i}\right)=\left\langle n_{i \sigma}\right\rangle$ for the noninteracting initial states at $\beta=10$ in the rotationally symmetric trap A (a) and the elongated trap B (b). In both cases, the total particle number per spin is $\left\langle N_{\sigma}\right\rangle=20\left(\mu_{\mathrm{A}}=3.146\right.$ and $\left.\mu_{\mathrm{B}}=4.515\right)$. The violet contour lines denote equipotential curves of the timeindependent harmonic confinement $V_{i}$.

the time grid we choose $N=200$ time steps on the time interval $\left[0, t_{\max }\right]=[0,10]$. Within the auxiliary bath scheme, convergence is obtained with $L=64$ bath orbitals at each site of the $10 \times 10$ lattice, i.e., the dimension of the associated single-particle Hilbert space is $D=10^{2}(1+64)=6500$. An efficient time stepping requires the storage of the auxiliary Hamiltonian in sparse matrix form [Eqs. (31) and (32)] on all time steps, i.e., approximately $6600 \times 200=1320000$ complex numbers. In contrast, the conventional solution of the Dyson equation would require storing the full Green function for 100 inequivalent sites and 200 time steps, which amounts to $100^{2} \times 200^{2}=400000000$ complex numbers, taking into account all Hermitian symmetries of Eq. (25).

\section{B. Time evolution of the density profile and double occupation}

For times $t \leqslant 0$, i.e., before the switch-on of any interactions, the systems $\mathrm{A}$ and $\mathrm{B}$ are characterized by equilibrium density matrices of the form

$$
\begin{aligned}
\rho_{j i \sigma}(0) & =\left\langle c_{i \sigma}^{\dagger}(0) c_{j \sigma}(0)\right\rangle \\
& =\sum_{\alpha}\langle i \sigma \mid \alpha\rangle\langle\alpha \mid j \sigma\rangle f_{\beta}\left(\epsilon_{\alpha}-\mu\right),
\end{aligned}
$$

where $\epsilon_{\alpha}$ and $\langle i \sigma \mid \alpha\rangle$ denote the eigenvalues and eigenvectors of the associated single-particle Hamiltonian [i.e., the matrix elements of Eq. (1) for $U=0$ ], and $f_{\beta}$ is the Fermi-Dirac distribution. In Fig. 6, we show the resulting density profiles $n_{\sigma}\left(\mathbf{R}_{i}\right)=\left\langle n_{i \sigma}\right\rangle=\rho_{i i \sigma}(0)$, which are centrally symmetric. The density of system $\mathrm{A}$ is in addition invariant under rotations of angle $\pi / 2$ due to the equal transverse confinements.

For $t>0$, the ramp of the Hubbard interaction $U$ drives the electrons in the traps A and B out of equilibrium. After the quench, i.e., when $U(t)$ has reached the stationary value $U_{\mathrm{f}}$, both systems evolve unitarily under a new and time-independent Hamiltonian $H^{\prime}=H\left(t_{\mathrm{q}}\right)$. In the course of this, they start to redistribute density and double occupation. Figures 7 and 8 show the time evolution of the local densities $\left\langle n_{i \sigma}\right\rangle(t)$ at all sites [see panels (a)] as well as the total double occupation $\langle d\rangle(t)=\frac{1}{\left\langle N_{\sigma}\right\rangle} \sum_{i}\left\langle d_{i}\right\rangle(t)$ [see panels (b)], obtained for traps $\mathrm{A}$ and $\mathrm{B}$ at $U_{\mathrm{f}}=2.0$. In addition, in panels (c) we 

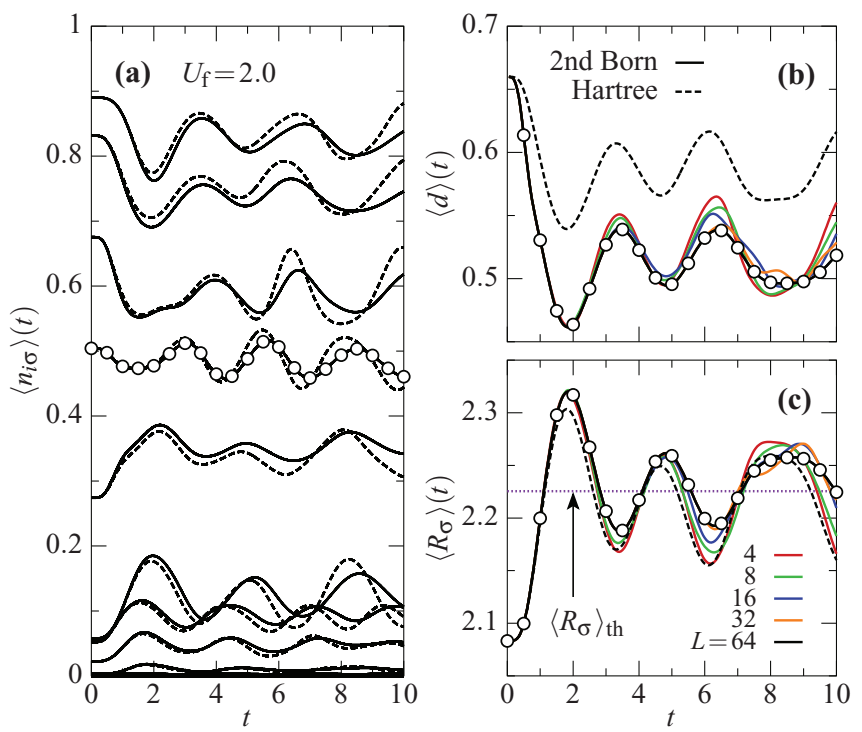

FIG. 7. (Color online) Time-dependent observables for the rotationally symmetric trap (system A) and $U_{\mathrm{f}}=2.0$. (a) site occupations $\left\langle n_{i \sigma}\right\rangle(t)$, (b) total double occupation $\langle d\rangle(t)$, and (c) radius $\left\langle R_{\sigma}\right\rangle(t)$ of the density profile. The black solid (dashed) lines show the result of the second Born (Hartree) approximation. The colored curves indicate the convergence of the results with the size $L$ of the bath in the auxiliary model (7). In panel (c), the violet dotted line refers to the radius $\left\langle R_{\sigma}\right\rangle_{\mathrm{th}}$ of an associated thermal equilibrium state (cf. Sec. IV B for discussion).

plot the time-dependent radius of the density profile $\left\langle R_{\sigma}\right\rangle(t)$ which is defined by

$$
\left\langle R_{\sigma}\right\rangle^{2}(t)=\frac{1}{\left\langle N_{\sigma}\right\rangle} \sum_{i}\left\langle n_{i \sigma}\right\rangle(t) \mathbf{R}_{i}^{2} .
$$

In Fig. 7(a) we can see (for system A) that immediately after the quench the atom cloud spreads out; sites of initially high density close to the trap center are depopulated and sites of initially low density at the boundary are populated, while densities closer to half filling exhibit comparatively smaller changes (open dots). Thereafter, the dynamics becomes oscillatory with clearly more than one frequency, which shows that the system is in a highly excited state after the interaction quench. In Fig. 8(a), we identify a similar behavior for the system B. In comparison to system A, some of the degeneracies are lifted such that the dynamics of the individual densities $\left\langle n_{i \sigma}\right\rangle(t)$ becomes more diverse. In addition, the increased confinement strength in the direction of $\omega_{2}$ leads to faster oscillations [compare also $\left\langle R_{\sigma}\right\rangle(t)$ and $\langle d\rangle(t)$ in Figs. 7 and 8] and to a nonuniform redistribution of density. The broadening of the density distribution and the subsequent collective oscillation are also well described by the time-dependent radius $\left\langle R_{\sigma}\right\rangle(t)$; see panels (c) in Figs. 7 and 8. Along with the initial expansion of the density, the double occupation decreases in both systems, cf. Figs. 7(b) and 8(b).

In Figs. 7 and 8 we have also included results obtained within the mean-field (Hartree) approximation (black dashed lines). The differences between the Hartree and the second Born approximation are more pronounced in $\langle d\rangle(t)$ than in
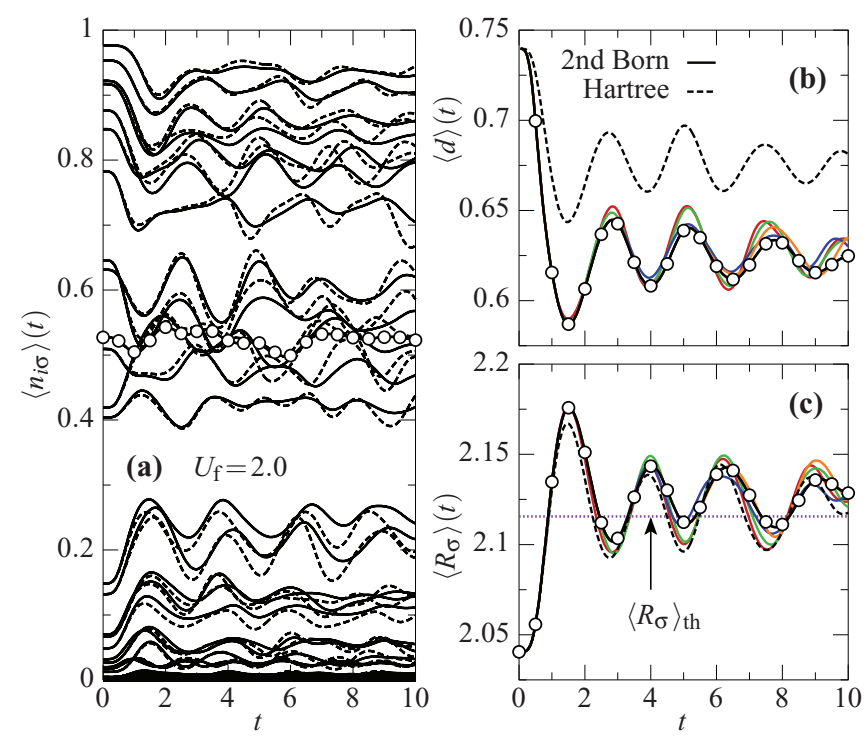

FIG. 8. (Color online) Same as Fig. 7 but for the elongated trap (system B). The final interaction strength is $U_{\mathrm{f}}=2.0$.

$\left\langle n_{i \sigma}\right\rangle(t)$, while both approximations lead to similar oscillations in the double occupation for times $t>2.0$.

In summary, the fast initial change and subsequent oscillations of all observables show that both systems A and B are not rapidly thermalizing. However, persistent oscillations make it hard to identify a prethermalization behavior, and it would be useful to find observables that can show signatures of a possible two-stage relaxation in a more clear-cut way, even for a small and confined system.

\section{Signatures of prethermalization in orbital occupations}

In a homogeneous system, the momentum occupations $n(\boldsymbol{k})$ provide the clearest evidence of prethermalization, through the discontinuity at the Fermi energy. Yet, for a small system with harmonic confinement, the momentum occupations follow a similar diverse and oscillating behavior as the real-space densities shown in Figs. 7 and 8, and a discontinuity in $n(\boldsymbol{k})$ is absent in the spatially inhomogeneous system even at temperature $T=0$. Therefore, a similar analysis of the two-stage relaxation as for the homogeneous case is rather difficult for the present systems. On the other hand, regarding the initial state of the system at $U=0$, one would still have a discontinuity in the occupations of the single-particle eigenfunctions $|\alpha\rangle$ of the trapped system [cf. Eq. (40)]. This fact motivates us to study the relaxation in terms of quantities that are more closely related to these natural orbitals of the system.

From the nonequilibrium Green function $G_{i j \sigma}\left(t, t^{\prime}\right)$ of the system, the time-dependent distribution function of any given orbital $|\alpha\rangle$ is accessible by

$$
f_{\alpha}(t)=-i \sum_{i j}\langle\alpha \mid i \sigma\rangle G_{i j \sigma}^{<}(t, t)\langle j \sigma \mid \alpha\rangle .
$$

In the following, we compare two different natural choices for $|\alpha\rangle$, which we refer to as the "initial-state basis" and the "final-state basis". The former is simply given by the 

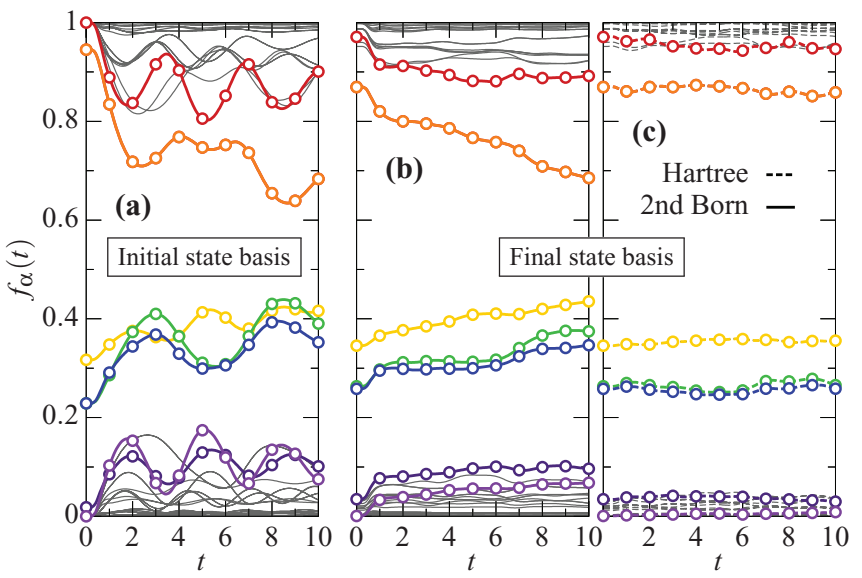

FIG. 9. (Color online) Time-dependent occupations $f_{\alpha}(t)$ for the "initial-state basis" (a) and the "final-state basis" (b) for the rotationally symmetric trap A and $U_{\mathrm{f}}=2.0$. Panel (c) shows the time evolution of $f_{\alpha}(t)$ for the final-state basis obtained within the Hartree approximation (see main text).

eigenfunctions of the noninteracting (initial) single-particle Hamiltonian $h_{i j}=\delta_{\langle i j\rangle} J_{0}+\left(V_{i}-\mu\right) \delta_{i j}$. The final-state basis will be defined by the eigenbasis of the mean-field Hamiltonian $\left(h_{\mathrm{th}}^{\sigma}\right)_{i j}=h_{i j}+U_{\mathrm{f}}\left(\left\langle n_{i \bar{\sigma}}\right\rangle+\frac{1}{2}\right) \delta_{i j}$, where the effective mean-field temperature $\beta_{\text {th }}$ is computed from the thermal equilibrium Hartree solution which has the same energy and particle number as the final state defined by the Green function $G_{i j \sigma}\left(t, t^{\prime}\right)$ for $t, t^{\prime} \geqslant t_{\mathrm{q}}$. The corresponding effective temperatures are $\beta_{\mathrm{th}}=3.20$ and $\beta_{\mathrm{th}}=4.05$ for the quench to $U_{\mathrm{f}}=2.0$ in systems A and $\mathrm{B}$, respectively (the adjusted chemical potentials are given in Figs. 10 and 11). Our choice of the single-particle states above is simply motivated by analogy to the homogeneous case, where both choices correspond to the plane-wave momentum states $|\boldsymbol{k}\rangle$ which well characterize the prethermalization behavior.

We first analyze the dynamics of the occupations for the rotationally symmetric trap A. Figures 9(a) and 9(b) show the time-dependent occupations of the initial-state basis and the final-state basis, respectively. As expected, most occupations correspond to orbitals $|\alpha\rangle$ that are either fully occupied $\left(f_{\alpha}=1\right)$ or almost empty $\left(f_{\alpha}=0\right)$ in the initial state. The most pronounced time-dependent changes are observed for orbitals close to the Fermi energy (bold colored curves). We find that the occupations of the initial-state basis still reflect the density oscillations shown in Figs. 7(a) and 7(c). The occupations of the finial-state basis, on the other hand, quite clearly reveal the two-stage relaxation: A rapid change of all time-dependent occupations $f_{\alpha}(t)$ on the time scale of a few inverse hoppings [see Fig. 9(b) for times $t \lesssim 2.0$ ] is followed by an almost monotonous drift at longer times [for the form of the prethermal distribution as function of the orbital energy; see Fig. 10(c)].

It would now be interesting to see whether the drift at long times corresponds to a true thermalization of the system. To this end, we in principle would need to compute the (final) interacting equilibrium state with the same amount of excitation energy. For an inhomogeneous system this is quite cumbersome, because multiple calculations are needed to find
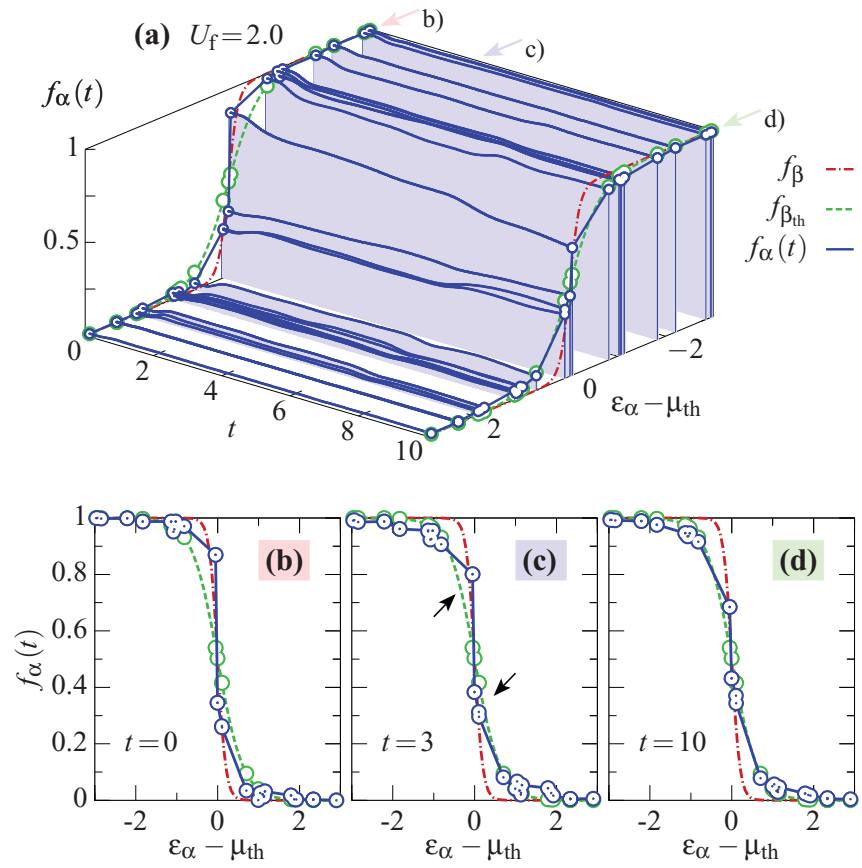

FIG. 10. (Color online) Relaxation dynamics in the rotationally symmetric trap A for a final Coulomb interaction of $U_{\mathrm{f}}=2.0$. (a) Time evolution of the distribution $f_{\alpha}(t)$ for the final-state basis (blue lines and dots) where $\beta_{\text {th }}=3.20$ and $\mu_{\text {th }}=-0.1093$. Panels (b)-(d) show cuts through the distribution of panel (a) at times $t=0$, 3.0, and 10. In all panels, the green dashed line shows the Fermi distribution $f_{\beta_{\mathrm{th}}}\left(\epsilon_{\alpha}^{\mathrm{th}}\right)$ in the final-state basis. As a guide for the eye, we also plot the Fermi distribution $f_{\beta}$ with temperature $\beta=10$ which characterizes the initial state at time $t=0$ (red dash-dotted curve).

the effective temperature $\beta_{\text {th }}$ at the correct chemical potential. On the other hand, for small values of $U_{\mathrm{f}}$, a mean-field description is usually still quite accurate for equilibrium states, even though higher-order scattering terms are of course crucial to correctly describe the actual relaxation dynamics to the thermalized state (this is in line with a description by kinetic equations, which reveals thermalization to a thermal state of the noninteracting system [7]). For this reason, it is worthwhile to compare the long-time behavior of the orbital occupations $f_{\alpha}$ to their values in the thermalized mean-field state, which by construction follow a Fermi distribution $f_{\beta_{\text {th }}}\left(\epsilon_{\alpha}^{\text {th }}\right)$ at effective temperature $1 / \beta_{\mathrm{th}}$. Figures 10(a)-10(d) plot the occupations in the final-state basis against time and the orbital energy $\epsilon_{\alpha}^{\text {th }}$. One can see that the drift of the occupations $f_{\alpha}(t)$ for times $t \gtrsim 3.0$ corresponds to a relaxation towards a thermalized state [see in particular the change of the occupations close to the Fermi energy from Fig. 10(c) (black arrows) to Fig. 10(d)]. This second relaxation process is harder to infer from observables discussed in Sec. IV B, even taking into account observables that involve averaging over the full trap. If we compare, e.g., the time evolution of the radius $\left\langle R_{\sigma}\right\rangle(t)$ in the system $\mathrm{A}$ to the thermal value $\left\langle R_{\sigma}\right\rangle_{\text {th }}$ [see the dotted lines in Fig. 7(c)], we observe an oscillation about this value but no clear evidence of damping.

For the elongated trap (system B), we find a very similar time dependence of the distribution function $f_{\alpha}(t)$; see Fig. 11 . 


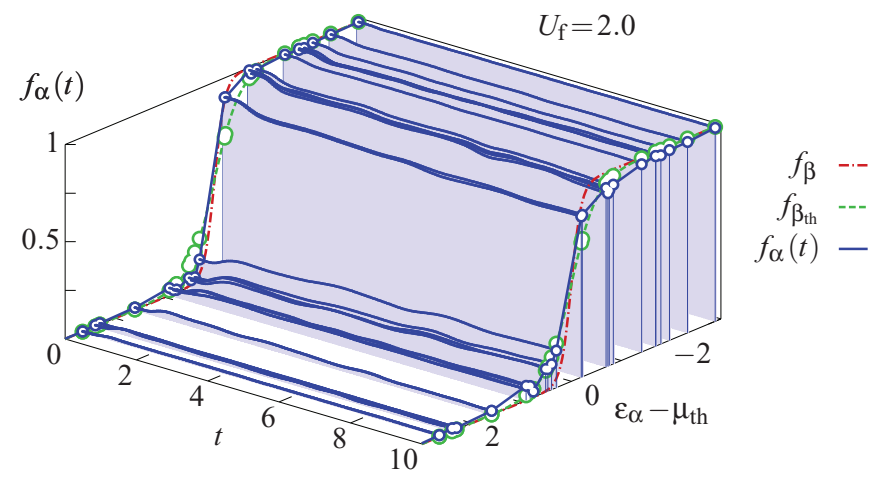

FIG. 11. (Color online) Same as in Fig. 10(a) but for the elongated trap B at $U_{\mathrm{f}}=2.0$. The effective temperature and the chemical potential in the final state basis are $\beta_{\mathrm{th}}=4.05$ and $\mu_{\mathrm{th}}=-0.0026$, respectively.

Although there happen to be no single-particle energy levels $\epsilon_{\alpha}$ very close to the Fermi edge, we can identify again an intermediate state which the system approaches on a similarly fast time scale, and further relaxation towards $f_{\beta_{\mathrm{th}}}$ at longer times.

In conclusion, we interpret the presence of the intermediate distributions $f_{\alpha}$ in the final-state basis around $t=3.0$ as a signature that the finite systems $\mathrm{A}$ and $\mathrm{B}$ prethermalize before they actually start to thermalize on a much longer time scale. That this prethermalization is mostly driven by correlations is demonstrated in Fig. 9(c) where we plot $f_{\alpha}(t)$ for the system $\mathrm{A}$ at $U_{\mathrm{f}}=2.0$ in Hartree approximation. In contrast to the calculation performed in the second-order approximation, the mean-field calculation leads to an almost stationary distribution $f_{\alpha}(t)$, even though the redistribution of the density $\left\langle n_{i \sigma}\right\rangle(t)$ as discussed in Sec. IVB is very similar in the Hartree and second Born approximation on the considered time interval [cf. Figs. 7(a) and 7(c)]. Finally, we mention that the two-stage relaxation is also obtained from the time-dependent eigenvalues of the density matrix $\rho_{i j \sigma}(t)$. The corresponding orbitals are the true natural orbitals of the system and interpolate between the initial- and final-state basis.

\section{SUMMARY}

In the present paper, we have formulated a method for solving the Dyson equation for an interacting quantum many-body system far from equilibrium [Eq. (2)] which avoids explicit memory integrations (or inversions of real time matrices). Instead, the approach maps local correlations to an auxiliary bath with finitely many orbitals. The problem of computing the Green function for the interacting many-body system is thereby reduced to an effective single-particle problem or, in other words, to an auxiliary Dyson equation which obeys purely Markovian instead of non-Markovian dynamics.

In Sec. II, we have presented the formalism in detail for self-energies which are local in space (single-site DMFT). The computational benefits of the method are however expected to carry over for a generalization to self-energies in cluster DMFT [38] or cluster perturbation theory [17]. In order to represent a nonlocal self-energy, the additional bath orbitals would be coupled to more than one site of the lattice, but the resulting Hamiltonian can still have a simple structure provided that the self-energy is sufficiently local in space. Furthermore, we note that although we have presented only calculations which start from noninteracting thermal states, the approach can easily be generalized to correlated initial states. The fundamentals of such an extension are formulated in [24] and lead to the inclusion of further sets of bath orbitals in Hamiltonian (7) which then mimic the decay of initial-state correlations.

In the context of DMFT, the auxiliary bath approach is most beneficial for lattice systems which are strongly inhomogeneous in space. In particular, it has enabled us to study an interaction quench for Fermions in an optical lattice, using inhomogeneous DMFT with second-order perturbation theory as an impurity solver (without a massive parallelization). We found that signatures of a two stage relaxation (prethermalization followed by slow thermalization) can be identified in the time-dependent occupations of single-particle orbitals which characterize the corresponding thermodynamic equilibrium state, although other observables like the local densities exhibits pronounced density oscillations after the quench. As an obvious extension of this work, one could further substantiate these results with more accurate impurity solvers, and study similar questions in the strong-coupling regime.

From the computational point of view, the efficiency of the auxiliary bath approach partially relies on the fact that self-energy decomposition can be more compact than the conventional representation on an equidistant time mesh. More precisely, our analysis in Sec. III has shown that the number of bath sites can typically be chosen smaller than the number of time steps which are propagated. Together with the fact that the auxiliary Hamiltonian is anyway very sparse when the self-energy is local, the compact representation of the self-energy leads over to a tremendous saving of computer memory when instead of the full Green function only the time-dependent parameters of the auxiliary model are stored.

Future applications of the method may consider more complicated self-energies (e.g., the $T$ matrix) or the usage of a strong-coupling impurity solver. Furthermore, it will be interesting to investigate decomposition schemes different from the Cholesky decomposition, in order to optimize the representation of the self-energy. In this sense, the auxiliary bath provides a starting point to address the issue of systematically truncating memory effects in the Dyson equation.

\section{ACKNOWLEDGMENTS}

We thank C. Gramsch, M. Kollar, M. Potthoff, and P. Werner for valuable discussions. Calculations have been performed at the PHYSnet computer cluster at University Hamburg.

\section{APPENDIX A: TIME PROPAGATION OF THE AUXILIARY GREEN FUNCTION}

For the second-quantized quadratic auxiliary Hamiltonian $H_{\mathrm{aux}}(t)=\sum_{i j \sigma} h_{\mathrm{aux}, i j}^{\sigma}(t) c_{i \sigma}^{\dagger} c_{j \sigma}$, where $h_{\mathrm{aux}, i j}^{\sigma}(t)$ is given by Eq. (30) and the indices $i, j \in\{1, \ldots, D\}$ run over lattice and bath sites [this is in contrast to the notation in Eq. (7) where we explicitly distinguish between bath and lattice creation and 
annihilation operators], we need to compute the lesser and greater components of the nonequilibrium Green function,

$$
G_{i j \sigma}^{\mathrm{aux}}\left(t, t^{\prime}\right)=-i\left\langle T_{\mathcal{C}} c_{i \sigma}(t) c_{j \sigma}^{\dagger}\left(t^{\prime}\right)\right\rangle_{\mathrm{aux}} .
$$

To derive an appropriate time-stepping algorithm, we start from the Heisenberg equations of motion for the creation and annihilation operators,

$$
\begin{aligned}
& i \partial_{t} c_{i \sigma}(t)=\left[c_{i \sigma}(t), H_{\mathrm{aux}}(t)\right]_{-}, \\
& i \partial_{t} c_{i \sigma}^{\dagger}(t)=\left[c_{i \sigma}^{\dagger}(t), H_{\mathrm{aux}}(t)\right]_{-},
\end{aligned}
$$

which in matrix form have the formal solutions

$$
C_{\sigma}(t)=U_{\sigma}(t, 0) C_{\sigma}(0), \quad C_{\sigma}^{\dagger}(t)=C_{\sigma}^{\dagger}(0) U_{\sigma}^{\dagger}(t, 0) .
$$

Here, the quantities $C_{\sigma}$ and $C_{\sigma}^{\dagger}$ are row and column vectors of the form $C_{\sigma}(t)=\left[c_{1 \sigma}(t), \ldots, c_{D \sigma}(t)\right]$ and $C_{\sigma}^{\dagger}(t)=$ $\left[c_{1 \sigma}^{\dagger}(t), \ldots, c_{D \sigma}^{\dagger}(t)\right]$, and $U_{\sigma}$ denotes the unitary time evolution operator

$$
U_{\sigma}\left(t^{\prime}, t\right)=T_{\mathrm{t}} \exp \left(-i \int_{t}^{t^{\prime}} d s h_{\mathrm{aux}}^{\sigma}(s)\right),
$$

with the usual time-ordering operator $T_{\mathrm{t}}$. If the initial state at time $t=0$ is described by the one-particle density matrix $\rho_{\sigma}^{\text {aux }}(0)=\left\langle C_{\sigma}^{\dagger}(0) C_{\sigma}(0)\right\rangle_{\text {aux }}$ which is symmetric, the lesser and greater components of the auxiliary Green function can be computed from

$$
\left[G_{\sigma}^{\mathrm{aux}}\right]^{\gtrless}\left(t, t^{\prime}\right)=\mp i U_{\sigma}(t, 0) R_{\sigma}^{\gtrless} U_{\sigma}^{\dagger}\left(t^{\prime}, 0\right),
$$

where $R_{\sigma}^{>}=1-\rho_{\sigma}^{\text {aux }}(0)$ and $R_{\sigma}^{<}=\rho_{\sigma}^{\text {aux }}(0)$ (note that 1 indicates the identity matrix here). Using the propagator property of $U_{\sigma}$, we can rewrite Eq. (A5) as

$$
\begin{aligned}
{\left[G_{\sigma}^{\mathrm{aux}}\right]^{\gtrless}\left(t, t^{\prime}\right) } & =\mp i U_{\sigma}(t, 0) R_{\sigma}^{\gtrless} U_{\sigma}^{\dagger}(s, 0) U_{\sigma}^{\dagger}\left(t^{\prime}, s\right) \\
& =\left[G_{\sigma}^{\mathrm{aux}}\right]^{\gtrless}(t, s) U_{\sigma}^{\dagger}\left(t^{\prime}, s\right)
\end{aligned}
$$

or

$$
\begin{aligned}
{\left[G_{\sigma}^{\mathrm{aux}}\right]^{\gtrless}\left(t, t^{\prime}\right) } & =\mp i U_{\sigma}(t, s) U_{\sigma}(s, 0) R_{\sigma}^{\gtrless} U_{\sigma}^{\dagger}\left(t^{\prime}, 0\right) \\
& =U_{\sigma}(t, s)\left[G_{\sigma}^{\mathrm{aux}}\right] \gtrless\left(s, t^{\prime}\right) .
\end{aligned}
$$

Hence, if we choose to propagate the greater correlations function $G_{\sigma}^{>}\left(t_{n}, t_{n^{\prime}}\right)$ for times $t_{n}>t_{n^{\prime}}$ (we omit the index "aux" for simplicity) and the lesser correlation functions $G_{\sigma}^{<}\left(t_{n}, t_{n^{\prime}}\right)$ for times $t_{n} \leqslant t_{n^{\prime}}$, where $n, n^{\prime} \in\{0,1,2, \ldots, N\}$ and $t_{0}=0$, the algorithm involves the following steps on each time slice $n$ $(m \leqslant n)[32]:$

$$
\begin{aligned}
G_{\sigma}^{>}\left(t_{n+1}, t_{m}\right) & =U_{\sigma}\left(t_{n+1}, t_{n}\right) G_{\sigma}^{>}\left(t_{n}, t_{m}\right), \\
G_{\sigma}^{<}\left(t_{m}, t_{n+1}\right) & =G_{\sigma}^{<}\left(t_{m}, t_{n}\right) U_{\sigma}^{\dagger}\left(t_{n+1}, t_{n}\right), \\
G_{\sigma}^{<}\left(t_{n+1}, t_{n+1}\right) & =U_{\sigma}\left(t_{n+1}, t_{n}\right) G_{\sigma}^{<}\left(t_{n}, t_{n}\right) U_{\sigma}^{\dagger}\left(t_{n+1}, t_{n}\right) .
\end{aligned}
$$

Note that on the time diagonal it is $G_{\sigma}^{>}\left(t_{n}, t_{n}\right)=-i+$ $G_{\sigma}^{<}\left(t_{n}, t_{n}\right)$. To establish the self-consistency directly on the time slice $n$, we further update the time evolution operator $U_{\sigma}\left(t_{n+1}, t_{n}\right)$ a few times by recalculating the single-particle Hamiltonian $h_{\text {aux }}^{\sigma}$ at the intermediate time $t_{n}+\frac{\Delta t}{2}$. This of course requires a few (low-rank) Cholesky decompositions of the self-energy.

\section{APPENDIX B: KRYLOV METHOD}

In order to adopt a Krylov-based time propagation scheme [39] to Eqs. (A8a), (A8b), and (A8c), we split the matrix multiplications $U_{\sigma} G_{\sigma}^{>}$and $G_{\sigma}^{<} U^{\dagger}=\left[U_{\sigma}\left(G_{\sigma}^{<}\right)^{\dagger}\right]^{\dagger}$ into sets of matrix-vector multiplications of the form

$$
U_{\sigma} G_{\sigma}=U_{\sigma}\left(G_{1 \sigma}, \ldots, G_{D \sigma}\right),
$$

where $G_{i \sigma}$ denotes the $i$ th column of the matrix $G_{\sigma}$ which is either $G_{\sigma}^{>}$or $\left(G_{\sigma}^{<}\right)^{\dagger}$. For a small time step $\Delta t \ll 1$, each product $U_{\sigma}(t+\Delta t, t) G_{i \sigma}$ can then be evaluated by applying the Krylov method [40],

$$
\begin{aligned}
U_{\sigma}(t+\Delta t, t) G_{i \sigma} & =\left|G_{i \sigma}\right| \exp \left\{-i h_{\text {aux }}^{\sigma}\left(t+\frac{\Delta t}{2}\right) \Delta t\right\} \frac{G_{i \sigma}}{\left|G_{i \sigma}\right|} \\
& \approx\left|G_{i \sigma}\right| V_{\sigma}^{(M)} \exp \left\{-i H_{\sigma}^{(M)} \Delta t\right\} e_{1}^{(M)}, \quad \text { (B2) }
\end{aligned}
$$

where it is essential to first normalize the vectors $G_{i \sigma}$. In the last line of Eq. (B2), the matrix $V_{\sigma}^{(M)}=\left(V_{1 \sigma}, \ldots, V_{M \sigma}\right)$ is of dimension $D \times M$ and contains an orthonormal basis of the Krylov space,

$$
\mathcal{K}^{(M)}=\operatorname{span}\left(v, h_{\sigma} v, h_{\sigma}^{2} v, \ldots, h_{\sigma}^{M-1} v\right),
$$

where $v=G_{\sigma i}$ and $h_{\sigma}=h_{\text {aux }}^{\sigma}\left(t+\frac{\Delta t}{2}\right)$. Further,

$$
H_{\sigma}^{(M)}=\left[V_{\sigma}^{(M)}\right]^{*} h_{\sigma} V_{\sigma}^{(M)}
$$

is a tridiagonal matrix of dimension $M \times M$ which can easily be diagonalized, and $e_{1}^{(M)}$ denotes the first unit vector in $\mathbb{R}^{M}$. In all practical calculations, a sufficient accuracy is obtained for $M \ll D$.

Finally, we emphasize that the solution of the original lattice problem (1) requires the computation of the auxiliary Green function $G_{i j \sigma}^{\mathrm{aux}}\left(t, t^{\prime}\right)$ only for indices $i, j$ which are lattice (and not bath) indices; see Eq. (9). This can be exploited to further simplify the time propagation. More precisely, it allows one to evolve $\left[G_{\sigma}^{\mathrm{aux}}\right]^{<}\left(\left[G_{\sigma}^{\mathrm{aux}}\right]^{>}\right)$away from the time diagonal only for those rows (columns) which involve lattice indices, cf. Eqs. (A8a) and (A8b). Along the time diagonal, such a simplification is inhibited by the specific structure of Eq. (A8c) which requires the knowledge of all matrix elements of the Green function. Furthermore, the time propagation is easily parallelized by performing the independent matrix-vector multiplications in Eq. (B1) simultaneously on many CPUs.
[1] S. Wall, D. Brida, S. R. Clark, H.P. Ehrke, D. Jaksch, A. Ardavan, S. Bonora, H. Uemura, Y. Takahashi, T. Hasegawa, H. Okamoto, G. Cerullo, and A. Cavalleri, Nat. Phys. 7, 114 (2011).
[2] D. Fausti, R. I. Tobey, N. Dean, S. Kaiser, A. Dienst, M. C. Hoffmann, S. Pyon, T. Takayama, H. Takagi, and A. Cavalleri, Science 331, 189 (2011). 
[3] A. Kirilyuk, A. V. Kimel, and T. Rasing, Rev. Mod. Phys. 82, 2731 (2010).

[4] I. Bloch, J. Dalibard, and W. Zwerger, Rev. Mod. Phys. 80, 885 (2008).

[5] U. Schneider, L. Hackermüller, J. P. Ronzheimer, S. Will, S. Braun, T. Best, I. Bloch, E. Demler, S. Mandt, D. Rasch, and A. Rosch, Nat. Phys. 8, 213 (2012).

[6] R. Sensarma, D. Pekker, E. Altman, E. Demler, N. Strohmaier, D. Greif, R. Jördens, L. Tarruell, H. Moritz, and T. Esslinger, Phys. Rev. B 82, 224302 (2010).

[7] M. Stark and M. Kollar, arXiv:1308.1610.

[8] M. Moeckel and S. Kehrein, Phys. Rev. Lett. 100, 175702 (2008).

[9] J. Berges, S. Borsányi, and C. Wetterich, Phys. Rev. Lett. 93, 142002 (2004).

[10] M. Eckstein, M. Kollar, and P. Werner, Phys. Rev. Lett. 103, 056403 (2009).

[11] M. Schiró and M. Fabrizio, Phys. Rev. Lett. 105, 076401 (2010).

[12] B. Sciolla and G. Biroli, Phys. Rev. Lett. 105, 220401 (2010).

[13] N. Tsuji, M. Eckstein, and P. Werner, Phys. Rev. Lett. 110, 136404 (2013).

[14] J. K. Freericks, V. M. Turkowski, and V. Zlatic, Phys. Rev. Lett. 97, 266408 (2006).

[15] H. Aoki, N. Tsuji, M. Eckstein, M. Kollar, T. Oka, and P. Werner, arXiv:1310.5329 [Rev. Mod. Phys. (to be published)].

[16] K. Mikelsons, J. K. Freericks, and H. R. Krishnamurthy, Phys. Rev. Lett. 109, 260402 (2012).

[17] M. Balzer and M. Potthoff, Phys. Rev. B 83, 195132 (2011).

[18] C. Jung, A. Lieder, S. Brener, H. Hafermann, B. Baxevanis, A. Chudnovskiy, A. Rubtsov, M. Katsnelson, and A. Lichtenstein, Ann. Phys. 524, 49 (2012).

[19] F. Hofmann, M. Eckstein, E. Arrigoni, and M. Potthoff, Phys. Rev. B 88, 165124 (2013).

[20] L.V. Keldysh, Sov. Phys. JETP 20, 1018 (1965) [Zh. Eksp. Teor. Fiz. 47, 1515 (1964)].

[21] L. P. Kadanoff and G. Baym, Quantum Statistical Mechanics (Benjamin, New York, 1962).
[22] K. Balzer, S. Bauch, and M. Bonitz, Phys. Rev. A 82, 033427 (2010).

[23] K. Balzer, S. Hermanns, and M. Bonitz, J. Phys.: Conf. Ser. 427, 012006 (2013).

[24] C. Gramsch, K. Balzer, M. Eckstein, and M. Kollar, Phys. Rev. B 88, 235106 (2013).

[25] G. Stefanucci and R. van Leeuwen, Nonequilibrium Many-Body Theory of Quantum Systems (Cambridge University Press, Cambridge, UK, 2013).

[26] K. Balzer and M. Bonitz, Nonequilibrium Green's Function Approach to Inhomogeneous Systems, Lecture Notes in Physics Vol. 867 (Springer, Heidelberg, 2013).

[27] G. Baym, Phys. Rev. 127, 1391 (1962).

[28] J. K. Freericks, Phys. Rev. B 77, 075109 (2008).

[29] P. Danielewicz, Ann. Phys. 152, 239 (1984).

[30] H. S. Köhler, N. H. Kwong, and H. A. Yousif, Comput. Phys. Commun. 123, 123 (1999).

[31] M.-T. Tran, Phys. Rev. B 78, 125103 (2008).

[32] A. Stan, N. E. Dahlen, and R. van Leeuwen, J. Chem. Phys. 130, 224101 (2009).

[33] M. Eckstein, M. Kollar, and P. Werner, Phys. Rev. B 81, 115131 (2010).

[34] M. Garny and M. M. Müller, High Performance Computing in Science and Engineering (Garching/Munich, Springer, Berlin, 2010).

[35] C. Kollath, A. M. Läuchli, and E. Altman, Phys. Rev. Lett. 98, 180601 (2007).

[36] M. Kollar and M. Eckstein, Phys. Rev. A 78, 013626 (2008).

[37] M. Kollar, F. A. Wolf, and M. Eckstein, Phys. Rev. B 84, 054304 (2011).

[38] T. Maier, M. Jarrell, T. Pruschke, and M. H. Hettler, Rev. Mod. Phys. 77, 1027 (2005).

[39] M. Balzer, N. Gdaniec, and M. Potthoff, J. Phys.: Condens. Matter 24, 035603 (2012).

[40] M. Hochbruck and C. Lubich, SIAM J. Numer. Anal. 34, 1911 (1997). 